\title{
STELLAR AND GAS PROPERTIES OF HIGH H I MASS-TO-LIGHT RATIO GALAXIES IN THE LOCAL UNIVERSE
}

\author{
Bradley E. Warren ${ }^{1}$ and Helmut JerJen \\ Research School of Astronomy and Astrophysics, Australian National University, Mount Stromlo Observatory, \\ Cotter Road, Weston, ACT 2611, Australia; bewarren@mso.anu.edu.au, jerjen@mso.anu.edu.au \\ AND \\ BäRBEL S. KORIBALSKI \\ Australia Telescope National Facility, CSIRO, P.O. Box 76, Epping, NSW 1710, Australia; baerbel.koribalski@csiro.au \\ Received 2005 September 25; accepted 2005 December 16
}

\begin{abstract}
We present a multiwavelength study (BVRI-band photometry and $\mathrm{H}$ I line interferometry) of nine late-type galaxies selected from the HIPASS Bright Galaxy Catalog on the basis of apparently high $\mathrm{H}$ I mass-to-light ratios $\left(3 \mathcal{M}_{\odot} / L_{\odot, B}<\mathcal{M}_{\mathrm{H}_{\mathrm{I}}} / L_{B}<27 \mathcal{M}_{\odot} / L_{\odot, B}\right)$. We find that most of the original estimates for $\mathcal{M}_{\mathrm{H}_{\mathrm{I}}} / L_{B}$ based on available photographic magnitudes in the literature were too high and conclude that genuine high $\mathrm{H}$ I mass-to-light ratio $\left(>5 \mathcal{M}_{\odot} / L_{\odot, B}\right)$ galaxies are rare in the local universe. Extreme high- $\mathcal{M}_{\mathrm{H}} / L_{B}$ galaxies such as ESO 215-G?009 appear to have formed only the minimum number of stars necessary to maintain the stability of their $\mathrm{H}$ I disks and could possibly be used to constrain galaxy formation models. They may also have been forming stars at a low, constant rate over their lifetimes. The best examples all have highly extended $\mathrm{H}$ I disks, are spatially isolated, and have normal baryonic content for their total masses but are deficient in stars. This suggests that high- $\mathcal{M}_{\mathrm{H}} / L_{B}$ galaxies are not lacking the baryons to create stars but are underluminous, as they lack either the internal or external stimulation for more extensive star formation.

Key words: galaxies: dwarf — galaxies: evolution — galaxies: individual (ATCA J061608-574552, ESO 121-G020, ESO 215-G?009, ESO 348-G009, ESO 428-G033, ESO 473-G024, ESO 505-G007, ESO 572-G009, IC 4212, MCG -04-02-003) — galaxies: irregular — galaxies: ISM galaxies: kinematics and dynamics - galaxies: photometry
\end{abstract}

\section{INTRODUCTION}

The number of low-mass dark matter halos predicted by models of a CDM (cold dark matter) dominated universe far exceeds the quantity of observed dwarf galaxies, typically by several orders of magnitude (see Kauffmann et al. 1993; Moore et al. 1999; Klypin et al. 1999). In this context we consider a galaxy to be a dark matter halo that contains baryons. Consequently, the slope of dark matter mass functions generally rises much more steeply than observed galaxy luminosity functions (Trentham \& Tully 2002; Hilker et al. 2003; Blanton et al. 2003; but see also Blanton et al. 2005).

While there are some physical processes that could help narrow this discrepancy (Klypin et al. 1999; Shapiro et al. 2004), the theoretical low-mass halo frequency is not reduced enough to reconcile them with observations. So we are left with the conclusions that either the current most favored cosmological models significantly overestimate the number of low-mass dark matter halos present in the local universe, or the observations have failed to find the vast majority of low-mass galaxies to date. If the latter were true, then it is important to look at why dwarf galaxies could be missed and how we could detect them.

Two reasons why galaxies might not have been found yet in optical surveys are that they could exhibit low stellar densities $\left(<1 \mathcal{M} \odot \mathrm{pc}^{-2}\right)$ or most of their baryons are in invisible form. In the extreme case, they do not contain any baryonic matter at all and are in fact "empty" dark matter halos. These possibilities could be dark matter halos in which the star formation from

\footnotetext{
${ }^{1}$ Affiliated with the Australia Telescope National Facility, CSIRO.
}

accreted gas has been halted, suppressed, or never began, or there were simply no baryons to form stars to begin with. Such objects lacking stars might be considered as being "dark galaxies," which could be easily missed in surveys biased toward optical wavelengths (Disney 1976). However, dark galaxies have yet to be found in the blind $\mathrm{H}$ I surveys such as the $\mathrm{H}$ I Parkes All-Sky Survey (HIPASS; Koribalski et al. 2004; Doyle et al. 2005).

If they do exist, large numbers of low-mass dark galaxies could naturally steepen the mass functions from observations, which are mostly derived from optical or near-infrared galaxy luminosity functions without consideration of other baryonic matter. Recent large-scale observations of neutral hydrogen gas ( $\mathrm{H} \mathrm{I}$ ) are now allowing mass functions to be derived based on nonstellar properties. Zwaan et al. (2003) produced one of the most extensive H I mass functions based on a catalog of the $1000 \mathrm{H}$ I-brightest galaxies in the southern hemisphere and found a low-mass end slope similar to that of other observational studies, again in contradiction with $\Lambda \mathrm{CDM}$ models.

Galaxies that have failed to convert most of their primordial gas into stars, and thus have retained a high proportion of $\mathrm{H} \mathrm{I}$, may well provide a partial solution. While not entirely "dark," these galaxies are hard to detect optically but may be detectable through $21 \mathrm{~cm}$ line observations. The $\mathrm{H}$ I mass-to-light ratio $\left(\mathcal{M}_{\mathrm{H}} / L_{B}\right.$, which compares the $\mathrm{H}$ I mass to the $B$-band luminosity) of these objects could be significantly higher than the typical ratios measured for late-type galaxies, so that they would be in a lower mass bin for a luminosity function than they would be for a baryonic mass function. If high- $\mathcal{M}_{\mathrm{H}} / L_{B}$ galaxies existed in significant numbers, then they could help correct the discrepancy in two ways, by including more previously unknown galaxies and 
by shifting known galaxies to higher mass bins than they would be placed in with purely optical results.

An example of an extreme $\mathrm{H}$ I mass-to-light ratio object, which could be described as a "dim" galaxy, is the nearby dwarf irregular ESO 215-G?009 with $\mathcal{M}_{\mathrm{H}_{\mathrm{I}}} / L_{B}=22 \pm 4 \mathcal{M}_{\odot} / L_{\odot, B}$ (Warren et al. 2004, hereafter Paper I). This faint low surface brightness dwarf irregular was found to be spatially isolated (1.7 Mpc from the nearest neighbor), with a low current star formation rate $\left(\lesssim 2.5 \times 10^{-3} \mathcal{M} \odot \mathrm{yr}^{-1}\right)$. It has an extended regularly rotating $\mathrm{H}$ I disk that can be traced out to over 6 times the Holmberg radius of the optical galaxy, making it one of the most extended $\mathrm{H}$ I envelopes relative to the optical extent.

Paper I included an analysis of the $\mathrm{H}$ I gas surface density of ESO 215-G?009. The azimuthally averaged surface density at all radii was below the critical gas surface density needed for largescale star formation as defined by the Toomre (1964) stability criteria (Kennicutt 1989; Martin \& Kennicutt 2001). It was proposed in Verde et al. (2002) that a large fraction of low-mass halos may form Toomre-stable gas disks and become "dark" galaxies, possibly $95 \%$ of objects with halo masses of $\lesssim 10^{10} \mathcal{M} \odot$. If so, ESO 215-G?009 would be just the tip of the iceberg. If we can find more galaxies similar to ESO 215-G?009, where the gas density after gravitational collapse is too low for efficient star formation, it may go some way to explaining the discrepancy between the dark matter halo mass function and the observed galaxy luminosity function.

To continue our study of the stellar and gas properties of galaxies with high $\mathcal{M}_{\mathrm{H}} / L_{B}$ values, we have selected a sample of nine galaxies from the Bright Galaxy Catalog (BGC) in the approximate range $3 \mathcal{M} \odot / L_{\odot, B}<\mathcal{M}_{\mathrm{H}} / L_{B}<27 \mathcal{M}_{\odot} / L_{\odot, B}$. In this paper, $\S 2$ looks at what was previously known about the sample galaxies. Section 3 summarizes our $21 \mathrm{~cm}$ and optical observations. Sections 4 and 5 present the results of the $\mathrm{H}_{\mathrm{I}}$ line observations and optical photometry, respectively. Section 6 compares the properties of individual galaxies. Section 7 contains the discussion of these results and the possible implications, while $\S 8$ gives our conclusions.

\section{GALAXY SELECTION}

The HIPASS BGC (Koribalski et al. 2004) lists the $1000 \mathrm{H} \mathrm{I-}$ brightest extragalactic sources (by $\mathrm{H}$ I peak flux density) in the southern hemisphere $\left(v_{\text {sys }}<8000 \mathrm{~km} \mathrm{~s}^{-1}\right)$. Photometric and structural parameters for the BGC's optical counterparts were obtained in 2002 from the Lyon-Meudon Extragalactic Database (LEDA; Paturel et al. 1997 and references therein; now moved to HyperLEDA) ${ }^{2}$ to study the statistical properties and various scaling relations of these galaxies. First estimates of $\mathrm{H}$ I mass-to-light ratios were obtained for 789 BGC galaxies that had mean apparent $B$-band photographic magnitudes from LEDA, using the equation

$$
\frac{\mathcal{M}_{\mathrm{H}_{\mathrm{I}}}}{L_{B}}=1.5 \times 10^{-7} F_{\mathrm{H}_{\mathrm{I}}} 10^{0.4\left(m_{B}-A_{B}\right)} \frac{\mathcal{M} \odot}{L_{\odot, B}},
$$

where $\mathcal{M}_{\mathrm{H}}$ is the $\mathrm{H}$ I mass in solar units, $L_{B}$ is the $B$-band luminosity in solar units, $F_{\mathrm{H}_{\mathrm{I}}}$ is the integrated $\mathrm{H}_{\mathrm{I}}$ flux density in Jy km s${ }^{-1}, m_{B}$ is the apparent $B$ magnitude, and $A_{B}$ is the $B$-band Galactic extinction. Extinction correction from the host galaxy is not included for reasons explained in $\S$ 7.2. Figure 1 shows the $\log \left(\mathcal{M}_{\mathrm{H}} / L_{B}\right)$ distribution for these galaxies as a function of their absolute $B$ magnitude:

$$
M_{B, 0}=m_{B}-A_{B}-5 \log D-25 \mathrm{mag},
$$

\footnotetext{
${ }^{2}$ See http://www-obs.univ-lyon1.fr/hypercat.
}

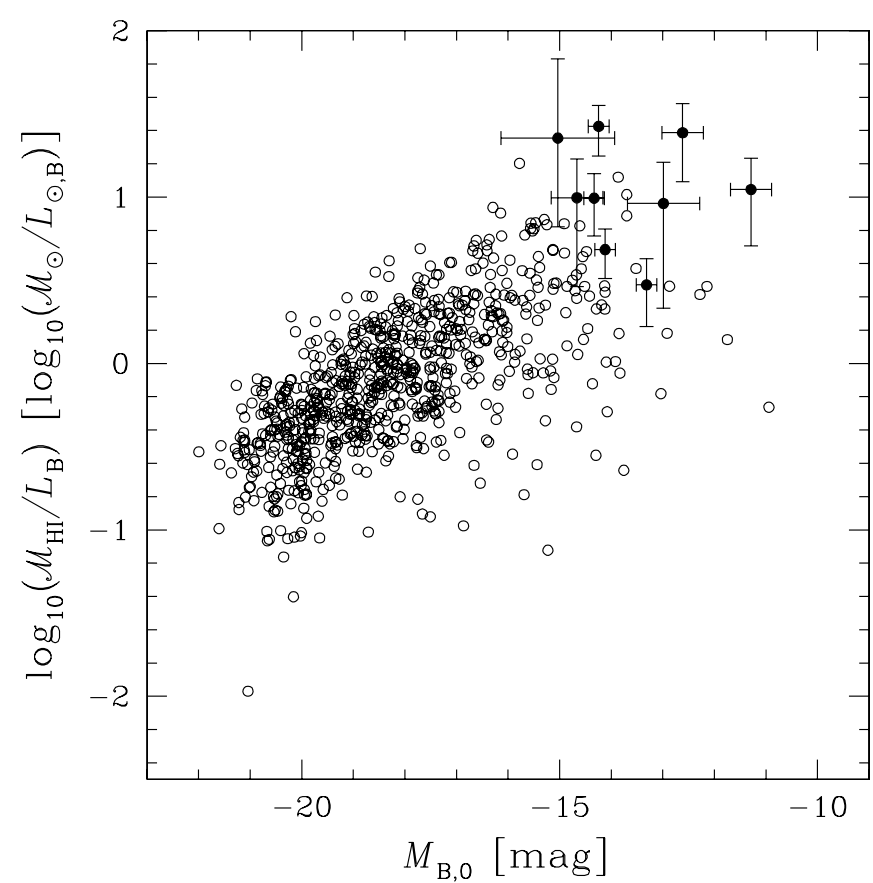

FIG. 1.-H I mass-to-light ratio vs. absolute photographic $B$ magnitude for 789 galaxies in the BGC that have blue apparent magnitudes listed in LEDA. The filled circles with error bars highlight the positions of the nine galaxies examined as part of this study.

where the galaxy distances $D(\mathrm{Mpc})$ were calculated from the Local Group velocities given in the BGC. Throughout this paper we adopt $H_{0}=75 \mathrm{~km} \mathrm{~s}^{-1} \mathrm{Mpc}^{-1}$.

The relation between these two quantities seems to suggest that many of the low-luminosity galaxies listed in the BGC have high $\mathcal{M}_{\mathrm{H}_{\mathrm{I}}} / L_{B}$ values, up to $27 \mathcal{M}_{\odot} / L_{\odot, B}$, well above typical values for late-type galaxies of less than $1 \mathcal{M} \odot / L_{\odot, B}$ (median $\mathcal{M}_{\mathrm{H}} / L_{B}$ of $0.78 \mathcal{M}_{\odot} / L_{\odot, B}$ in Roberts \& Haynes [1994], for type $\mathrm{Sm} / \mathrm{Im}$ galaxies). The nine filled circles mark the positions of the galaxies subject to our detailed follow-up observations, including ESO 215-G?009 (Paper I). The galaxies were chosen for various reasons, mostly because of a high estimated $\mathcal{M}_{\mathrm{H}} / L_{B}$ but also for reasons of unusual morphology, and after initial Australia Telescope Compact Array (ATCA) follow-up observations showed some unexpected results (see $\S 4.1$ ). Previously measured properties of those nine galaxies are summarized in Table 1.

\section{OBSERVATIONS}

Each galaxy was observed in two different wavelength regimes. Optical CCD photometry was obtained with the Australian National University $2.3 \mathrm{~m}$ telescope at the Siding Spring Observatory. H I $(21 \mathrm{~cm})$ line data were obtained with the ATCA.

\subsection{Radio Observations}

ATCA H I line observations of the selected galaxies were carried out between 2002 June and 2003 June. The galaxies ESO 121-G020, ESO 428-G033, and ESO 348-G009 $\left(\delta<-30^{\circ}\right)$ were observed for $2 \times \sim 12 \mathrm{hr}$ in different east-west arrays, while ESO 215-G?009 was observed for $3 \times \sim 12 \mathrm{hr}$. For the other five galaxies $\left(\delta>-30^{\circ}\right)$, we used the compact hybrid arrays that include antennas on the northern spur, resulting in rather large synthesized beams. The galaxies MCG -04-02-003, ESO 505G007, and IC 4212 were observed for $\sim 10 \mathrm{hr}$, while ESO 572G009 and ESO 473-G024 were only observed in snapshot mode ( $\sim 1-2 \mathrm{hr}$ taken over a $12 \mathrm{hr}$ period). H I snapshot observations 
TABLE

Summary of Previously Measured Galaxy Properties

\begin{tabular}{|c|c|c|c|c|c|c|c|c|c|c|c|c|}
\hline \multirow[b]{3}{*}{$\begin{array}{l}\text { NAME } \\
(1)\end{array}$} & \multirow[b]{3}{*}{$\begin{array}{c}\text { HIPASS NAME } \\
\text { (2) }\end{array}$} & \multirow{2}{*}{\multicolumn{2}{|c|}{ Center (J2000.0) }} & \multirow{3}{*}{$\begin{array}{c}l \\
(\mathrm{deg}) \\
(5)\end{array}$} & \multirow{3}{*}{$\begin{array}{c}b \\
(\mathrm{deg}) \\
(6)\end{array}$} & \multicolumn{3}{|c|}{ BGC } & \multirow{3}{*}{$\begin{array}{l}\text { LEDA } m_{B} \\
\quad(\mathrm{mag}) \\
(10)\end{array}$} & \multirow{3}{*}{$\begin{array}{c}\mathrm{SFD} 98 A_{B} \\
\text { (mag) } \\
(11)\end{array}$} & \multicolumn{2}{|c|}{ BGC + LEDA } \\
\hline & & & & & & & & & & & & \\
\hline & & $\begin{array}{c}\alpha \\
(3)\end{array}$ & $\begin{array}{c}\delta \\
(4)\end{array}$ & & & $\begin{array}{c}\left(\mathrm{km} \mathrm{s}^{-1}\right) \\
(7)\end{array}$ & $\begin{array}{l}(\mathrm{Mpc}) \\
(8)\end{array}$ & $\begin{array}{c}\left(\mathrm{Jy} \mathrm{km} \mathrm{s}{ }^{-1}\right) \\
(9)\end{array}$ & & & $\begin{array}{l}(\mathrm{mag}) \\
(12)\end{array}$ & $\begin{array}{c}\left(\mathcal{M}_{\odot} / L_{\odot, B}\right) \\
(13)\end{array}$ \\
\hline MCG -04-02-003 ............ & J0019-22 & 001911 & -224014 & 62.6 & -81.4 & $669 \pm 3$ & 9.5 & $16.0 \pm 2.5$ & $15.8 \pm 0.2$ & $0.08 \pm 0.01$ & $-14.1 \pm 0.2$ & $4.8 \pm 1.6$ \\
\hline ESO 473-G024 ................... & $\mathrm{J} 0031-22$ & 003123 & -224602 & 75.7 & -83.7 & $540 \pm 4$ & 7.6 & $7.2 \pm 1.8$ & $16.2 \pm 0.2$ & $0.08 \pm 0.01$ & $-13.3 \pm 0.2$ & $3.0 \pm 1.3$ \\
\hline ESO 121-G020 ................... & J0615-57 & 061553 & -574324 & 266.5 & -27.3 & $577 \pm 5$ & 4.1 & $14.1 \pm 2.9$ & $17.0 \pm 0.4$ & $0.17 \pm 0.03$ & $-11.3 \pm 0.4$ & $11 \pm 6$ \\
\hline 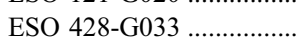 & $\mathrm{J} 0725-30 \mathrm{~B}$ & 072549 & -305505 & 244.2 & -6.9 & $1727 \pm 4$ & 19.5 & $12.8 \pm 2.7$ & $17.9 \pm 0.5$ & $1.10 \pm 0.18$ & $-14.7 \pm 0.5$ & $10 \pm 7$ \\
\hline ESO 215-G?009................... & $\mathrm{J} 1057-48$ & 105730 & -481102 & 284.1 & 10.5 & $598 \pm 2$ & 4.2 & $104.4 \pm 11.5$ & $16.4 \pm 0.4$ & $0.95 \pm 0.15$ & $-12.6 \pm 0.4$ & $24 \pm 12$ \\
\hline ESO 572-G009 .................... & $\mathrm{J} 1153-18$ & 115323 & -180959 & 284.1 & 42.6 & $1745 \pm 3$ & 20.4 & $8.5 \pm 1.9$ & $17.4 \pm 0.2$ & $0.16 \pm 0.03$ & $-14.3 \pm 0.2$ & $10 \pm 4$ \\
\hline ESO 505-G007 ..................... & $\mathrm{J} 1203-25$ & 120330 & -252822 & 289.5 & 36.1 & $1785 \pm 4$ & 20.8 & $20.5 \pm 3.2$ & $17.7 \pm 0.2$ & $0.36 \pm 0.06$ & $-14.2 \pm 0.2$ & $27 \pm 9$ \\
\hline 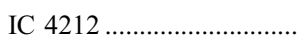 & $\mathrm{J} 1311-06$ & 131209 & -065831 & 312.0 & 55.5 & $1484 \pm 2$ & 18.1 & $47.5 \pm 4.6$ & $16.4 \pm 1.1$ & $0.19 \pm 0.03$ & $-15.0 \pm 1.1$ & $23_{-16}^{+45}$ \\
\hline 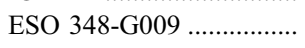 & $\mathrm{J} 2349-37$ & 234923 & -374623 & 349.8 & -73.2 & $648 \pm 4$ & 8.4 & $13.4 \pm 2.2$ & $16.7 \pm 0.7$ & $0.06 \pm 0.01$ & $-13.0 \pm 0.7$ & $9 \pm 7$ \\
\hline
\end{tabular}

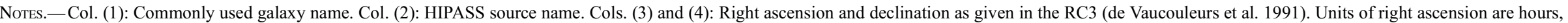

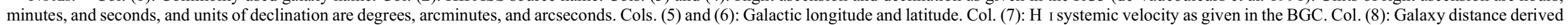

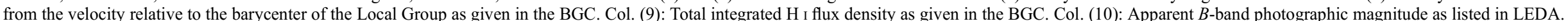

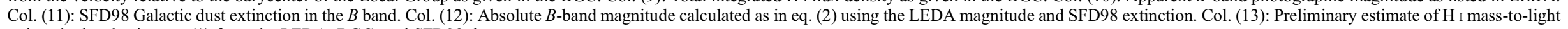
ratio calculated using eq. (1) from the LEDA, BGC, and SFD98 data 
TABLE 2

Summary of Radio Observations for Each Galaxy

\begin{tabular}{|c|c|c|c|c|}
\hline $\begin{array}{l}\text { Name } \\
\text { (1) }\end{array}$ & $\begin{array}{l}\text { Array } \\
(2)\end{array}$ & $\begin{array}{c}\text { Time on Source } \\
\text { (hr) } \\
\text { (3) }\end{array}$ & $\begin{array}{l}\text { Central Frequency } \\
(\mathrm{MHz}) \\
(4)\end{array}$ & $\begin{array}{c}\text { Phase Calibrator } \\
\text { (5) }\end{array}$ \\
\hline \multirow[t]{2}{*}{ MCG -04-02-003 ................... } & H75B & $\sim 1.5$ & 1417 & PKS $0023-263$ \\
\hline & H168B & $\sim 8.6$ & 1417 & PKS $0023-263$ \\
\hline \multirow[t]{2}{*}{ ESO 473-G024 ....................... } & $\mathrm{H} 75 \mathrm{~B}$ & $\sim 1.5$ & 1417 & PKS $0023-263$ \\
\hline & H168B & $\sim 1.0$ & 1417 & PKS $0023-263$ \\
\hline \multirow[t]{3}{*}{ ESO 121-G020 ............................. } & $750 \mathrm{D}$ & $\sim 10.5$ & 1417 & PKS 0407-658 \\
\hline & $1.5 \mathrm{~B}$ & $\sim 10.9$ & 1417 & PKS $0407-658$ \\
\hline & EW352 & $\sim 2.4$ & 1416 & PKS 0537-441 \\
\hline \multirow[t]{4}{*}{ 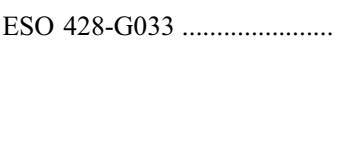 } & $750 \mathrm{D}$ & $\sim 10.6$ & 1412 & PKS 0614-349 \\
\hline & $1.5 \mathrm{~B}$ & $\sim 10.3$ & 1412 & PKS 0614-349 \\
\hline & EW352 & $\sim 1.1$ & 1414 & PKS 0614-349 \\
\hline & EW367B & $\sim 0.8$ & 1414 & PKS 0614-349 \\
\hline \multirow{3}{*}{ 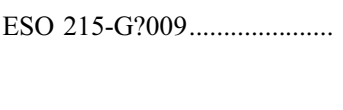 } & EW352 & $\sim 11.6$ & 1417 & PKS $1215-457$ \\
\hline & $750 \mathrm{~A}$ & $\sim 10.6$ & 1417 & PKS $1215-457$ \\
\hline & $6 \mathrm{~A}$ & $\sim 11.5$ & 1417 & PKS $1215-457$ \\
\hline ESO $572-G 009$ & $\mathrm{H} 75 \mathrm{~B}$ & $\sim 1.0$ & 1412 & PKS $1127-145$ \\
\hline \multirow[t]{3}{*}{ ESO 505-G007 . } & $\mathrm{H} 75 \mathrm{~B}$ & $\sim 1.0$ & 1412 & PKS $1127-145$ \\
\hline & H168B & $\sim 1.9$ & 1412 & PKS $1127-145$ \\
\hline & $\mathrm{H} 75 \mathrm{~B}$ & $\sim 9.2$ & 1412 & PKS $1151-348$ \\
\hline IC $4212 \ldots \ldots \ldots \ldots$ & $\mathrm{H} 75 \mathrm{~B}$ & $\sim 8.8$ & 1413 & PKS $1308-220$ \\
\hline \multirow[t]{3}{*}{ 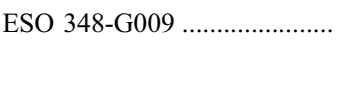 } & $750 \mathrm{D}$ & $\sim 10.6$ & 1417 & PKS $0008-421$ \\
\hline & $1.5 \mathrm{~B}$ & $\sim 9.8$ & 1417 & PKS $0008-421$ \\
\hline & EW352 & $\sim 1.7$ & 1417 & PKS $0008-421$ \\
\hline
\end{tabular}

Notes.-Col. (1): Galaxy name. Col. (2): ATCA configuration used. Col. (3): Approximate time on source for each array. Col. (4): Central observing frequency. Col. (5): Phase calibrator.

that were initially taken for the other galaxies (except ESO 215G?009 and IC 4212) were added to the other observations. Details of the $\mathrm{H}$ I observations for each galaxy are given in Table 2. We used a bandwidth of $8 \mathrm{MHz}$ divided into 512 channels, resulting in a channel width of $3.3 \mathrm{~km} \mathrm{~s}^{-1}$. The velocity resolution of the $\mathrm{H}$ I data is $\sim 4 \mathrm{~km} \mathrm{~s}^{-1}$. The primary calibrator for all observations was PKS 1934-638.

Data reduction and analysis were performed with the MIRIAD package using standard procedures, with further analysis using AIPS, GIPSY, and KARMA. Channels affected by Galactic H I emission were discarded where appropriate. After continuum subtraction, the H I data were Fourier-transformed using "natural" weighting and a channel width of $4 \mathrm{~km} \mathrm{~s}^{-1}$. The data were cleaned and restored with the synthesized beam (the size of which is given in Table 4 for each galaxy). Primary beam correction was applied. $\mathrm{H}$ I distributions (zeroth moment) were obtained for all galaxies using cutoffs between 3 and $4 \sigma$ and are shown in Figure 2, while the corresponding $\mathrm{H}$ I spectra are shown in Figure 3. For ESO 121G020, ESO 428-G033, ESO 215-G?009, and ESO 348-G009, mean velocity fields and dispersion maps were also produced, as they had sufficient resolution.

\subsection{Optical Photometry}

BVRI-band CCD images were obtained at the $2.3 \mathrm{~m}$ telescope as a series of 300 or $600 \mathrm{~s}$ exposures during observing runs between 2002 April and 2004 February using the Nasmyth Imager (SITe $1124 \times 1024$ thinned CCD). The imager has a circular field of view with a diameter of 6.62 and a pixel size of $0 .{ }^{\prime \prime} 59$. Table 3 gives a summary of the observations taken for each galaxy in each band. Most observations were taken at low air mass. Twilightsky flat fields in all bands and bias images were obtained at the same time. On each photometric night several Landolt (1992) standard stars were taken together with shallow $120 \mathrm{~s} B V R I$ im- ages of the galaxy fields to perform the photometric calibration of the deeper images.

Data reduction, photometric calibration, and analysis were carried out within IRAF using standard procedures. After overscan subtraction, bias subtraction, and flat-fielding, individual sets of images were registered and the sky level was subtracted. The images for each band were then combined into a single image (to increase the signal-to-noise ratio, remove cosmic rays, etc.), and the photometric calibration was applied. Figure 4 shows the resulting master images in the $B$ band for all nine galaxies.

Foreground stars were removed by replacing them with the surrounding sky so that only the galaxy remained. Special care was taken to restore the light distribution under any stars superposed onto the galaxies, e.g., using the mirror image from across the galaxies' center. For more details of this technique, see Jerjen (2003). To illustrate the final result, Figure 5 shows the $B$-band images of the galaxies after cleaning.

\section{RADIO PROPERTIES}

ATCA H I follow-up observations are needed to obtain accurate positions of the targeted HIPASS BGC sources and to reliably identify their optical counterparts. For those galaxies where we have high angular resolution $\mathrm{H}$ i observations (ESO 121-G020, ESO 428-G033, ESO 215-G?009, and ESO 348G009), we also analyze their $\mathrm{H}$ i structure and kinematics, including the galaxy rotation curve.

\subsection{H I Structure}

The $\mathrm{H}$ I distributions of all observed galaxies are shown in Figure 2 overlaid onto second-generation Digitized Sky Survey (DSS II) $R$-band images. The synthesized beam sizes for the individual observations differ significantly due to the use of either east-west or northern hybrid arrays. The ATCA H I spectra of the 
a) MCG-04-02-003

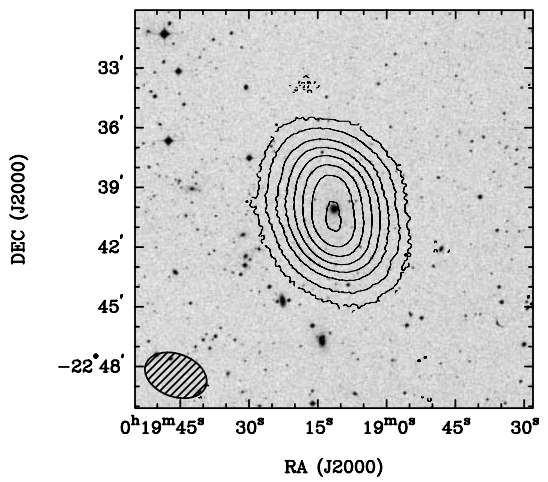

d) ESO 428-G033

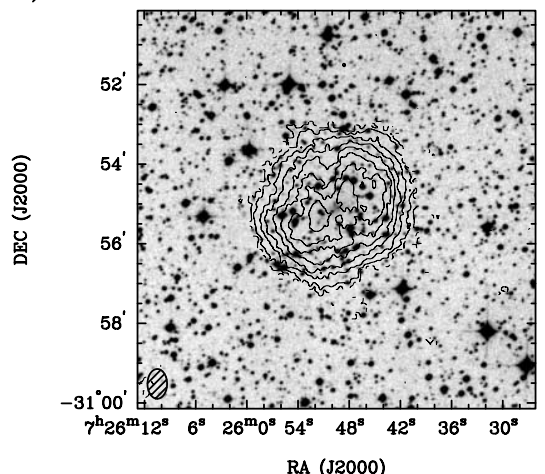

g) ESO 505-G007

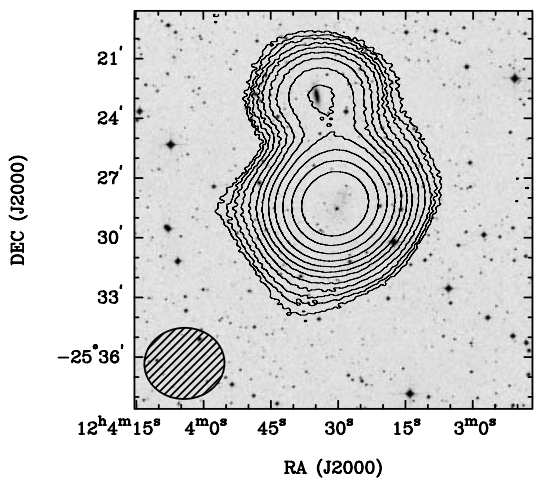

b) ESO 473-G024

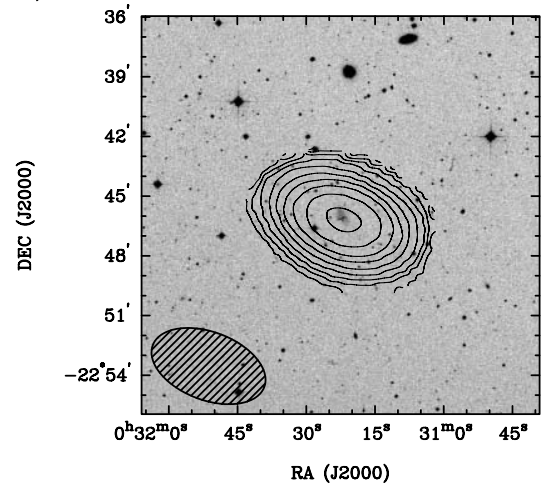

e) ESO 215-G?009

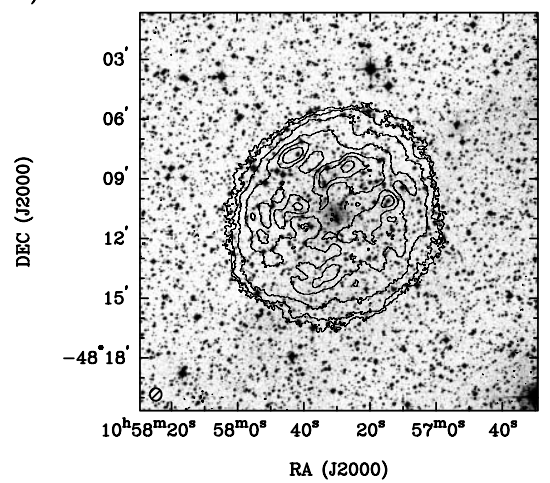

h) IC 4212

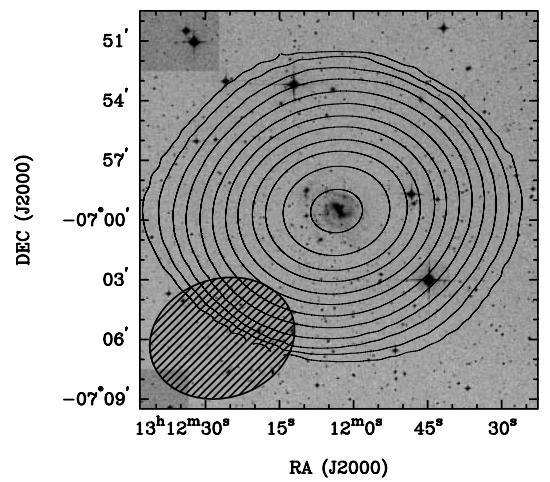

c) ESO 121-G020 \& ATCA J061608-574552
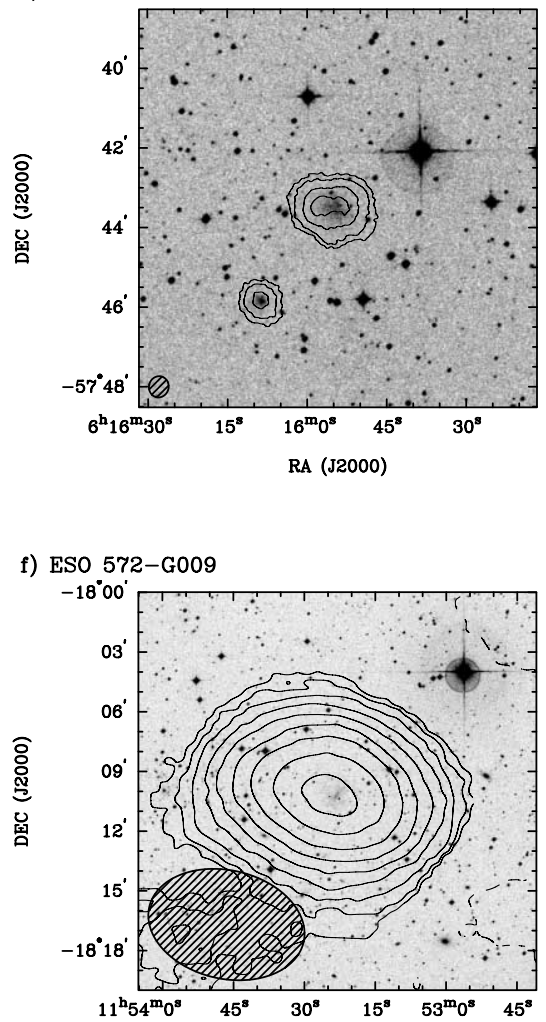

RA (J2000)

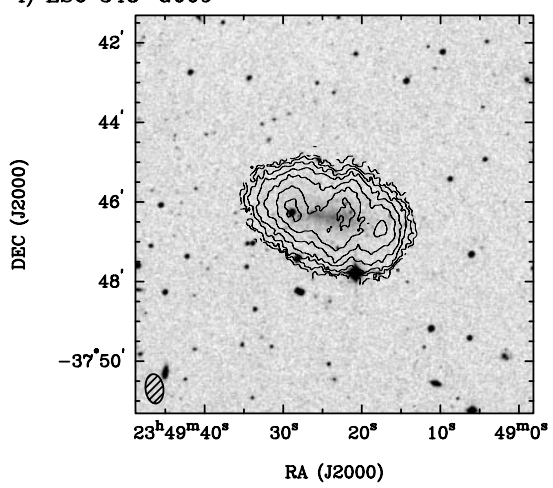

FIG. 2.-Integrated H I intensity distribution overlaid onto an optical DSS II $R$-band image. The panels show (a) MCG -04-02-003, $(b)$ ESO 473-G024, (c) ESO

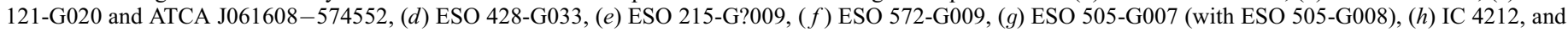
(i) ESO 348-G009. East is to the left and north is up for all images in this paper.

observed galaxies are shown in Figure 3, with the BGC spectra plotted for comparison.

The ATCA H I observations of the galaxy ESO 121-G020 reveal a previously uncataloged galaxy at a projected distance of only $3^{\prime}$ (see Fig. 2c). The two galaxies are well resolved spatially, and their systemic velocities differ by only $29 \pm 6 \mathrm{~km} \mathrm{~s}^{-1}$, less than the $50 \%$ velocity width of either galaxy. In the following we refer to this companion as ATCA J061608-574552 according to its $\mathrm{H}$ I center position. The $\mathrm{H}$ i spectra of the two galaxies, as well as the global spectrum of the galaxy pair, are shown in Figure 6 (Fig. $3 c$ shows the global spectrum only). ATCA J061608574552 has no previous optical measurements, and we included it in our optical follow-up observations (see $\S 5$ ). Because ATCA J061608-574552 contributes $\sim 20 \%$ to the $\mathrm{H}$ I flux density of HIPASS J0615-57, the H I mass-to-light ratio for ESO 121-G020 decreases slightly.
The galaxy ESO 505-G007 likewise has a neighbor, the cataloged galaxy ESO 505-G008, which lies at a projected distance of $\sim 7^{\prime}$. However, the two galaxies are not entirely spatially or spectrally resolved in our $\mathrm{H}$ i observations. ESO 505-G007 appears to have a low inclination in the optical images, and its $\mathrm{H}$ I line is relatively narrow. In contrast, the galaxy ESO 505-G008 is seen close to edge-on and shows a broad $\mathrm{H}$ I line, as seen in Figure $3 g$. By fitting two point sources to the low-resolution $\mathrm{H} \mathrm{I}$ distribution, we determine an $\mathrm{H}$ I flux density of $21 \pm 3 \mathrm{Jy} \mathrm{km} \mathrm{s}^{-1}$ for ESO 505-G007, in excellent agreement with the BGC value for HIPASS J1203-25 despite the confusion, and $8 \pm 3 \mathrm{Jy} \mathrm{km} \mathrm{s}^{-1}$ for ESO 505-G008.

The ATCA H I maps of the other seven galaxies match the optical counterparts identified in the BGC. For MCG -04-02-003 (Fig. $2 a$ ) the $\mathrm{H}$ I distribution is extended north-south and aligned with the stellar distribution (see $\S 5$ ), although little other structure 

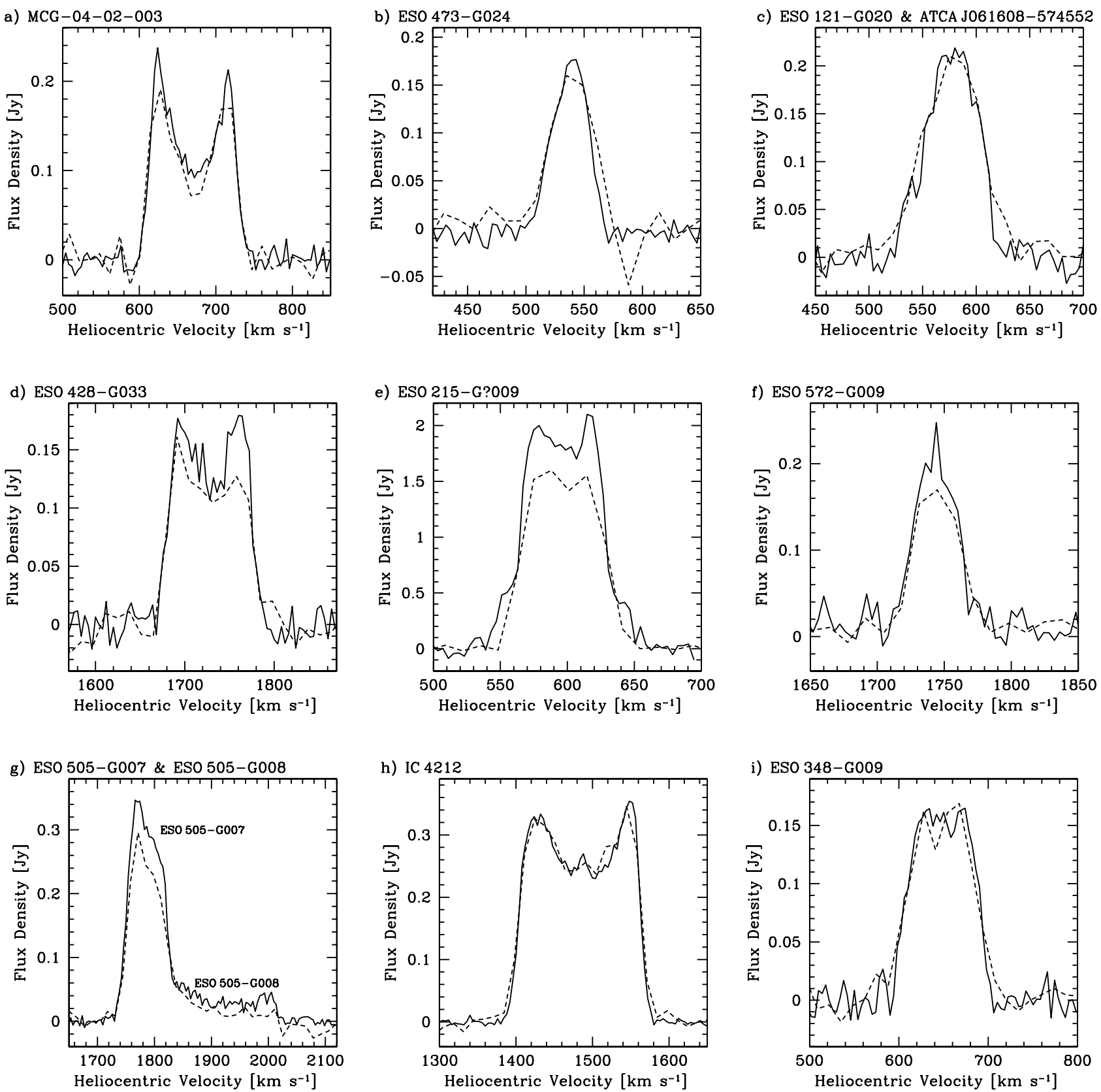

FIG. 3.-Global H I spectra of the nine galaxies as obtained from the BGC (dashed lines) and the ATCA (solid lines). The galaxies shown in the respective panels are as in Fig. 2.

is distinguishable with the large beam. For IC 4212 (Fig. $2 h$ ) the source appears extended compared to the large beam, and deconvolution with imfit in MIRIAD indicates that the $\mathrm{H}$ I extends $\sim 200^{\prime \prime}$. We find that for all galaxies except ESO 215-G?009 (see Paper I), the measured ATCA H I flux densities are in agreement with the BGC values (including the combined ESO 121-G020/ ATCA J061608-574552 system). Our results for the ATCA H I observations of the nine galaxies are listed in Table 4.

\subsection{H i Gas Dynamics}

The H I velocity fields (first moment) of the galaxies ESO 121G020/ATCA J061608-574552, ESO 428-G033, ESO 215-G?009 (Paper I), and ESO 348-G009 are shown in Figure 7. All galaxies show clear signs of rotation. The velocity field of the galaxy ESO 428-G033 appears similar to that of ESO 215-G?009, displaying fairly regular, undisturbed gas dynamics. The extreme velocity contours on both the approaching and receding sides close, sug- gesting that either the rotation curve turns down at large radii or the galaxy is warped. The position angle of ESO 428-G033, as measured from its $\mathrm{H}$ I velocity field, is aligned with the bright optical emission, although the nature of the stellar feature is unclear (see $\S$ 6.1.4). Likewise, the position angle of the galaxy ESO 348-G009 aligns well with the optical disk (see $\S \S 5$ and 6.1 for more on the optical details of the galaxies).

The analysis of the rotation curve for ESO 215-G?009 using rocur in AIPS was presented in Paper I. For the other three galaxies we instead used the equivalent procedure rotcur in GIPSY (both use the tilted-ring algorithm described by Begeman 1989). We used the standard procedure of narrowing the free parameters (center position, $v_{\text {sys }}$, position angle, and inclination) down one at a time until the best fit to all parameters was obtained and a rotation curve could be produced. All three fits were done with $10^{\prime \prime}$ rings (the ESO 215-G?009 fit used 12" rings). After an initial fit that included both sides of the galaxy, the fit was done 
TABLE 3

Summary of Optical Observations for Each Galaxy

\begin{tabular}{|c|c|c|c|}
\hline $\begin{array}{c}\text { Name } \\
\text { (1) }\end{array}$ & $\begin{array}{c}\text { Band } \\
\text { (2) }\end{array}$ & $\begin{array}{c}\text { Exposure Time } \\
\text { (s) } \\
\text { (3) }\end{array}$ & $\begin{array}{c}\text { Seeing } \\
\text { (arcsec) } \\
(4)\end{array}$ \\
\hline \multirow[t]{4}{*}{ MCG -04-02-003 ........... } & $B$ & $3000(10 \times 300)$ & 2.2 \\
\hline & $V$ & $2400(8 \times 300)$ & 2.0 \\
\hline & $R$ & $1800(6 \times 300)$ & 1.9 \\
\hline & $I$ & $1800(6 \times 300)$ & 2.2 \\
\hline \multirow[t]{4}{*}{ ESO 473-G024 .................. } & $B$ & $3000(10 \times 300)$ & 2.5 \\
\hline & $V$ & $2400(8 \times 300)$ & 3.3 \\
\hline & $R$ & $1800(6 \times 300)$ & 3.3 \\
\hline & $I$ & $1800(6 \times 300)$ & 2.0 \\
\hline \multirow[t]{2}{*}{ 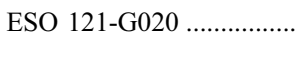 } & $B$ & $3000(10 \times 300)$ & 2.1 \\
\hline & $R$ & $1800(6 \times 300)$ & 1.9 \\
\hline \multirow[t]{4}{*}{ ESO 428-G033 …............ } & $B$ & $3000(5 \times 600)$ & 2.2 \\
\hline & $V$ & $2400(4 \times 600)$ & 2.0 \\
\hline & $R$ & $1800(3 \times 600)$ & 1.9 \\
\hline & $I$ & $1800(3 \times 600)$ & 3.0 \\
\hline \multirow[t]{4}{*}{ ESO $215-G ? 009 \ldots \ldots \ldots \ldots \ldots . . . . . .}$. & $B$ & $3000(10 \times 300)$ & 1.9 \\
\hline & $V$ & $2400(8 \times 300)$ & 1.9 \\
\hline & $R$ & $1800(6 \times 300)$ & 1.8 \\
\hline & $I$ & $1800(6 \times 300)$ & 2.0 \\
\hline \multirow[t]{3}{*}{ ESO $572-G 009$.................... } & $B$ & $1800(3 \times 600)$ & 1.9 \\
\hline & $V$ & $2400(8 \times 300)$ & 2.7 \\
\hline & $R$ & $1800(6 \times 300)$ & 2.2 \\
\hline \multirow[t]{3}{*}{ ESO 505-G007 …............... } & $B$ & $3000(10 \times 300)$ & 2.0 \\
\hline & $V$ & $1800(6 \times 300)$ & 2.2 \\
\hline & $R$ & $1800(6 \times 300)$ & 2.2 \\
\hline \multirow[t]{3}{*}{ 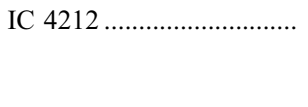 } & $B$ & $3000(5 \times 600)$ & 2.9 \\
\hline & $V$ & $2400(4 \times 600)$ & 2.3 \\
\hline & $R$ & $1800(3 \times 600)$ & 1.9 \\
\hline \multirow[t]{4}{*}{ ESO 348-G009 ................ } & $B$ & $3000(10 \times 300)$ & 1.7 \\
\hline & $V$ & $2400(8 \times 300)$ & 1.4 \\
\hline & $R$ & $1800(6 \times 300)$ & 1.5 \\
\hline & $I$ & $1800(6 \times 300)$ & 1.4 \\
\hline
\end{tabular}

Notes._Col. (1): Galaxy name. Col. (2): Broadband (Cousins) filters used. Col. (3): Total observing time in each of the optical bands, including the number of individual exposures. Col. (4): Atmospheric seeing in the final optical images.

individually for the approaching and receding sides of the galaxy to check for any asymmetry. Of the three new fits, the only galaxy that had significant differences between the two sides was ESO 428-G033, where the inclination fit was lower on the approaching side, which may have been a result of the very low inclination (this was included in the uncertainties).

The results offitting rotation curves to the $\mathrm{H}$ i velocity fields of these four galaxies are listed in Table 5. The final rotation curves are shown in Figure 8. The very low inclination of ESO 428G033 produced a high uncertainty in the rotation velocity values, as shown by the curves plotted. In the case of ESO 348-G009 the curve appears to still be rising at the last points of the rotation curve, suggesting that the $\mathrm{H}_{\mathrm{I}}$ is not tracing the galaxy out to the radius at which the maximum rotation velocity is reached (so $v_{\text {rot, max }}$ should be considered a lower limit in this case). This is similarly the case for ESO 121-G020, where we may just be reaching the point where the curve flattens out. In the other two galaxies we appear to have reached the flat part of the curve.

\section{OPTICAL PROPERTIES}

The observed $B$ images taken on the $2.3 \mathrm{~m}$ telescope of all nine galaxies are in Figure 4, while Figure 5 shows the same images after star subtraction. Star contamination is obviously a problem for ESO 215-G?009 and especially ESO 428-G033, both of which are close to the Galactic plane, so special care was taken with these images. Some of the galaxies (notably MCG - 04-02003, ESO 215-G?009, ESO 505-G007, and ESO 348-G009) were affected by moderately bright foreground stars sitting on top of the galaxy that had to be removed with care. As mentioned in $\S 4$, a companion galaxy to ESO 121-G020 was found in the $\mathrm{H}$ I imaging. The optical image for ESO 121-G020 also includes ATCA J061608-574552, and the photometry for that galaxy is included with the other results here. For each galaxy a growth curve was measured on the star-subtracted images from the luminosityweighted center in 2 pixel $(\sim 1$.' 2$)$ circular aperture rings to obtain the total intensity and a surface brightness profile. Our results for the optical BVRI photometry taken on the $2.3 \mathrm{~m}$ telescope are in Table 6.

Surface brightness profiles for the nine initially selected galaxies in each of the observed bands are in Figure 9, while the profile of ATCA J061608-574552 is in Figure 10. All profiles have been corrected for Galactic extinction, but no attempt to correct for inclination was made as it is difficult to calculate given the morphology of many galaxies, and as the correction for edge-on galaxies (such as ESO 348-G009) would be unrealistic without correction for the thickness of the stellar disk. The profiles for several galaxies show some of the underlying morphology. MCG -04-02-003 (Fig. 9a) has two distinct components, an inner bright bulge region that is prominent in the optical image, and a surrounding low surface brightness disk (almost 4 mag fainter in surface brightness). IC 4212 (Fig. 9h) appears to have a small central bulge, and the effects of the spiral arms are evident in the bumps of the profile. ESO 572-G009 (Fig. $9 f$ ) also seems to have a small central bulge, which is seen in the image (Fig. 5f ). ESO 348-G009 and ESO 428-G033 (Figs. 9d and 9i) exhibit pure exponential disks, while ESO 473-G024 (Fig. 9b) is similar but with a flatter central region.

The various light profiles of ESO 505-G007 (Fig. 9g) are flat in the central region, as the luminosity-weighted center of this galaxy is a faint region between many denser sites that are probably star formation regions. The optical structure of this galaxy (see Fig. $5 g$ ) possibly reflects a highly disturbed galaxy with increased star formation due to interaction with the neighboring galaxy ESO 505-G008 (the source that contaminates its $\mathrm{H}$ I spectrum). The new galaxy ATCA J061608-574552 notably has a much steeper exponential profile than its companion ESO 121-G020, as well as a higher central surface brightness. This denser structure may be the result of recent star formation (there is a bright region in the center of the galaxy that could be a star formation site) and may have led to its previously being missed in optical surveys if it were mistaken for a star (it is falsely identified as a star in the USNO star catalog; Monet et al. 2003).

For only two galaxies, ESO 473-G024 and ESO 215-G?009, did we find $B$-band apparent magnitudes that are in agreement with the values quoted in LEDA (even within their sometimes large error bars). In the case of all seven other galaxies, our value of $m_{B}$ was brighter than that given in LEDA, sometimes by several magnitudes. The effect this has on the $\mathrm{H}$ I mass-to-light ratios and why this occurred so often in our sample is discussed in the following sections ( $\S \S 6.1$ and 7.1).

\section{DISCUSSION OF INDIVIDUAL GALAXIES}

\subsection{Comparing Optical and $\mathrm{H}$ I Properties}

Now that we have more accurate optical and $\mathrm{H}$ I measurements we can recalculate many of the physical properties of the galaxies, including $\mathcal{M}_{\mathrm{H}} / L_{B}$. Table 7 summarizes new parameters from our observations. Values for $\mathcal{M}_{\text {tot }}, \mathcal{M}_{\mathrm{H}} / \mathcal{M}_{\text {tot }}$, and 
a) MCG-04-02-003
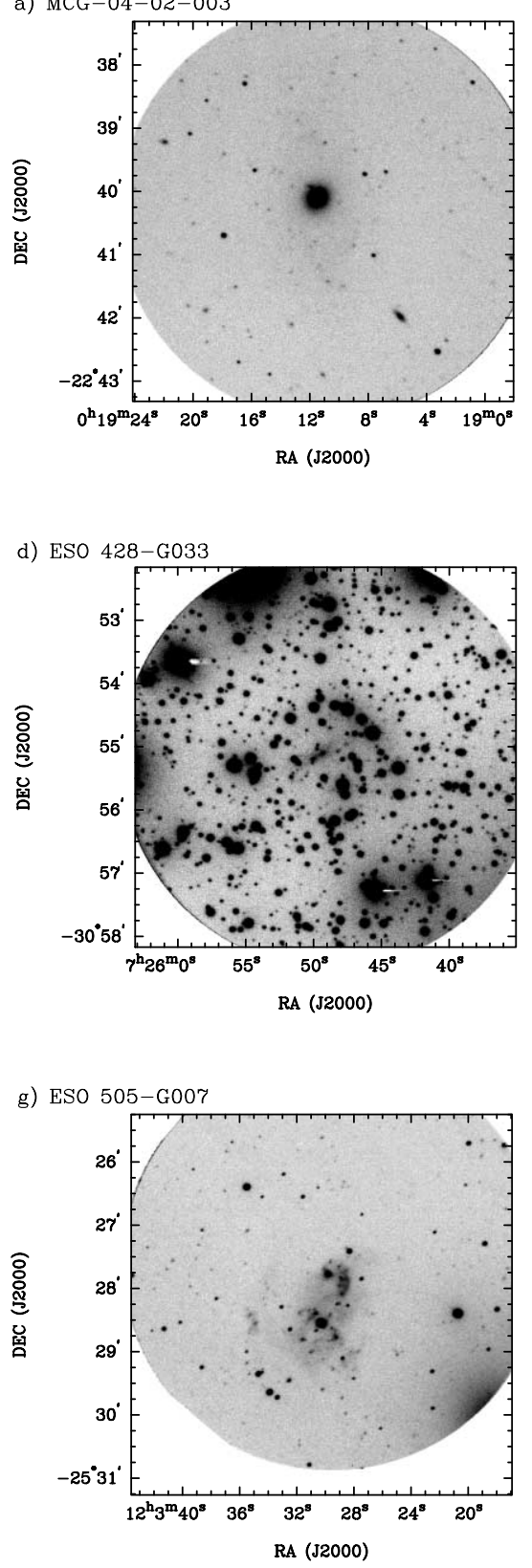

b) ESO $473-$ G024
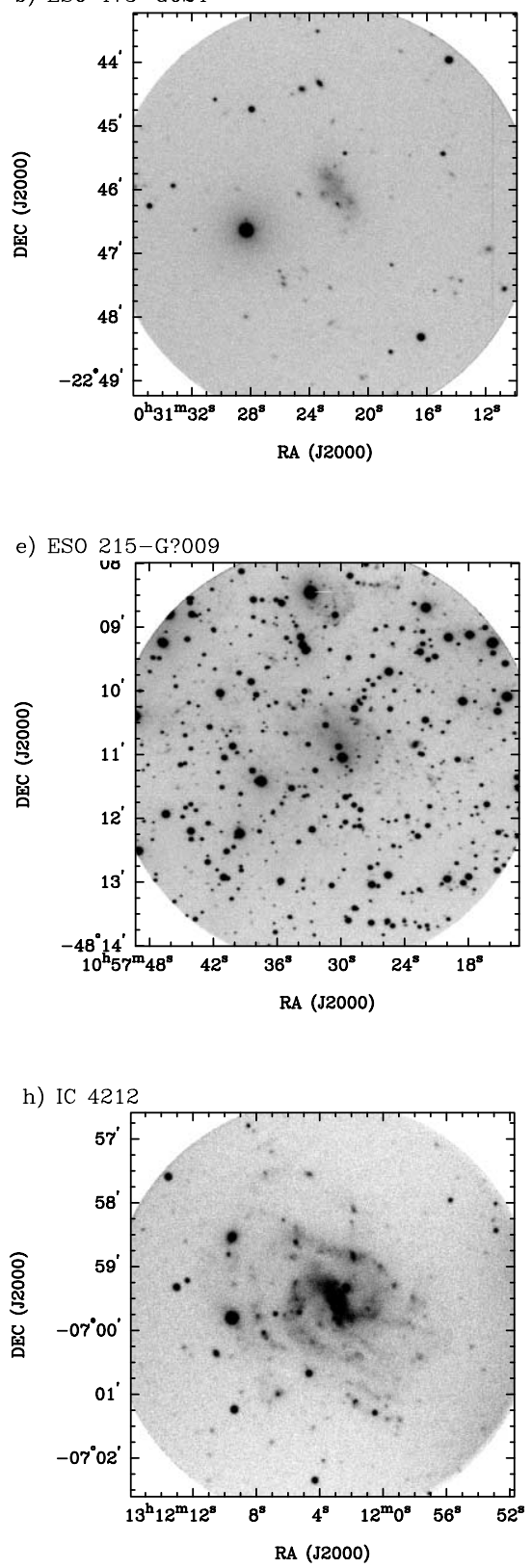

c) ESO 121-G020 \& ATCA J061608-574552
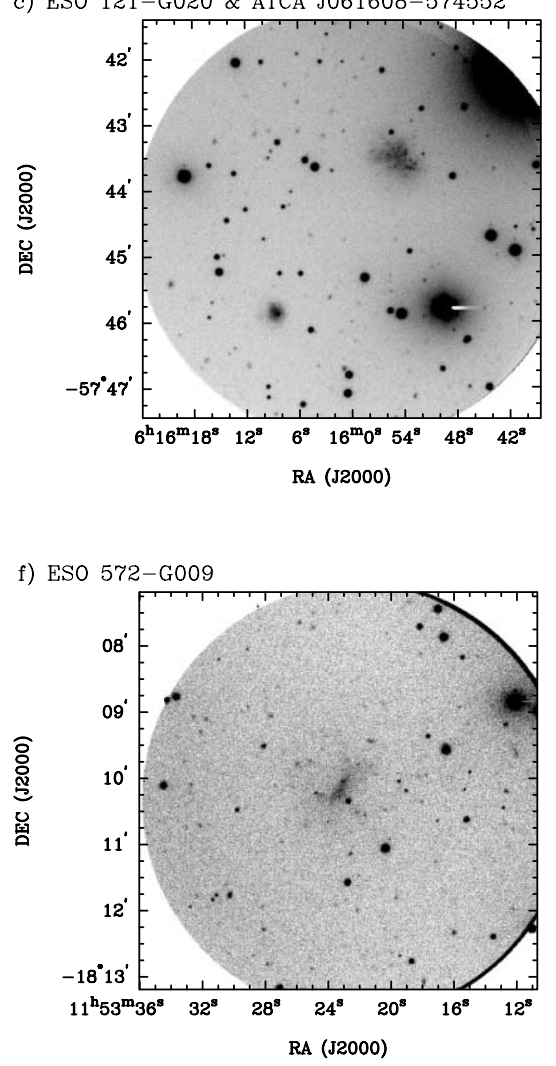

FIG. 4.- Observed deep $B$-band images of all galaxies. The galaxies shown in the respective panels are as in Fig. 2.

$\mathcal{M}_{\text {tot }} / L_{B}$ (cols. [6]-[8]) are only given for the four galaxies for which we fit rotation curves.

\subsection{1. $M C G-04-02-003$}

Our $B$-band apparent magnitude for this galaxy is $\sim 0.5 \mathrm{mag}$ brighter than that expressed in LEDA, which was based on the APM (Automatic Plate Measuring) plate scan results of Maddox et al. (1990). The galaxy has a distinctive optical morphology (Fig. 5a). A shallow CCD image shows only a small, bright, centrally concentrated circular region like a blue compact dwarf. However, deeper observations reveal a second, low surface brightness disk component extending well beyond this central bulge, which is clearly visible in our surface brightness profile for this galaxy (Fig. 9a). Difficulty in measuring the full extent of this faint disk could account for the disagreement between our measurement and the previous result of Maddox et al. (1990). The H I flux density result, on the other hand, is in excellent agreement with the
BGC and the observations of Fouque et al. (1990), suggesting we have recovered almost all the Parkes flux density. The result of our combined $\mathrm{H}_{\mathrm{I}}$ and optical values is that the $\mathrm{H}$ I mass-to-light ratio drops moderately to $3.0 \pm 0.3 \mathcal{M} \odot / L_{\odot, B}$, still higher than "typical" late-type galaxies but not unusually so. Despite the large beam for the MCG -04-02-003 observations, we can clearly see that the $\mathrm{H}$ I envelope is extended in a north-south direction. This is in the same direction as the outer low surface brightness optical disk, and the two correlate quite well in both shape and extent.

\subsubsection{ESO 473-G024}

Of the nine sample galaxies, ESO 473-G024 had the best agreement with the $B$-band apparent magnitude quoted by LEDA, also agreeing with the results in Lauberts \& Valentijn (1989) and Maddox et al. (1990). Our H I flux density is in agreement with both the BGC and Fouqué et al. (1990). Consequently, $\mathcal{M}_{\mathrm{H}} / L_{B}$ 

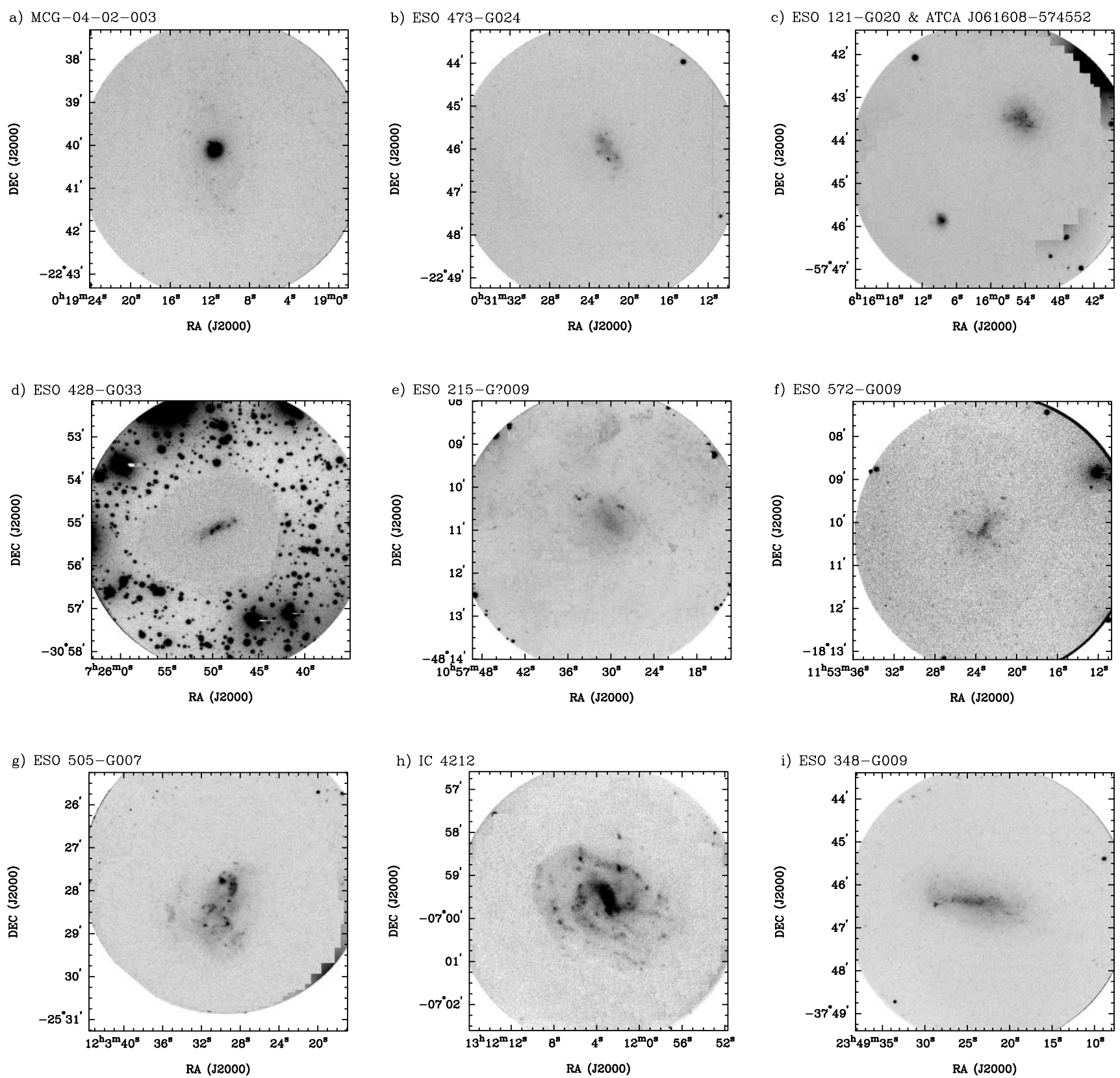

FIG. 5. $-B$-band images of all galaxies after the foreground stars subtraction process. The galaxies shown in the respective panels are as in Fig. 2.

remained at a moderate ratio of $2.8 \pm 0.6 \mathcal{M} \odot / L_{\odot, B}$, one of the higher ratios of this sample after accurate measurements. It is a well-studied dwarf irregular from the Sculptor group, and the $\mathcal{M}_{\mathrm{H}} / L_{B}$ value has been noted before (Skillman et al. 2003a, 2003b). In the optical the galaxy is extended in the north-south direction and has a number of potential star formation regions (see Fig. $5 b$ ), some of which were studied by Skillman et al. (2003a, 2003b; see also $\S 7.3$ ).

\subsubsection{ESO 121-G020 and ATCA J061608-574552}

The unexpected discovery of the companion galaxy ATCA J061608-574552 to the southeast of ESO 121-G020 must affect the $\mathcal{M}_{\mathrm{H}} / L_{B}$ value previously calculated for this galaxy, as the BGC H I flux density measurement for HIPASS J0615-57 includes the $\mathrm{H}$ i emission from both galaxies. But more importantly, our optical $B$ magnitude determined here is $\sim 1.7$ mag brighter than the value in LEDA, although it is closer to the Lauberts \&
Valentijn (1989) total magnitude (15.85 $\pm 0.09 \mathrm{mag})$. The new ratio for the combined system is $1.62 \pm 0.18 \mathcal{M} \odot / L_{\odot, B}$, and for the individual galaxies it is $1.50 \pm 0.13 \mathcal{M}_{\odot} / L_{\odot, B}$ for ESO 121G020 and $2.2 \pm 0.3 \mathcal{M} \odot / L_{\odot, B}$ for ATCA J061608-574552. Down to our sensitivity limits there is neither an $\mathrm{H}$ i nor a stellar bridge between the two galaxies detected.

\subsection{4. $\mathrm{ESO} 428-G 033$}

At only 6.9 from the Galactic plane, ESO 428-G033 suffers from relatively high Galactic extinction $(1.10 \pm 0.18 \mathrm{mag}$ in $B$; Schlegel et al. 1998) and foreground star contamination (see Fig. $4 d$ ). Our $B$-band magnitude of $16.90 \pm 0.10 \mathrm{mag}$ is $\sim 1 \mathrm{mag}$ brighter than the LEDA value (which has a high uncertainty of $0.5 \mathrm{mag}$ ) but agrees with the Lauberts \& Valentijn (1989) measurement $(16.83 \pm 0.09 \mathrm{mag})$. Despite this, with our new data the galaxy still has a high $\mathrm{H}$ I mass-to-light ratio of $4.5 \pm 0.9 \mathcal{M}_{\odot} / L_{\odot, B}$, which is in good agreement with the results of Kraan-Korteweg \& 


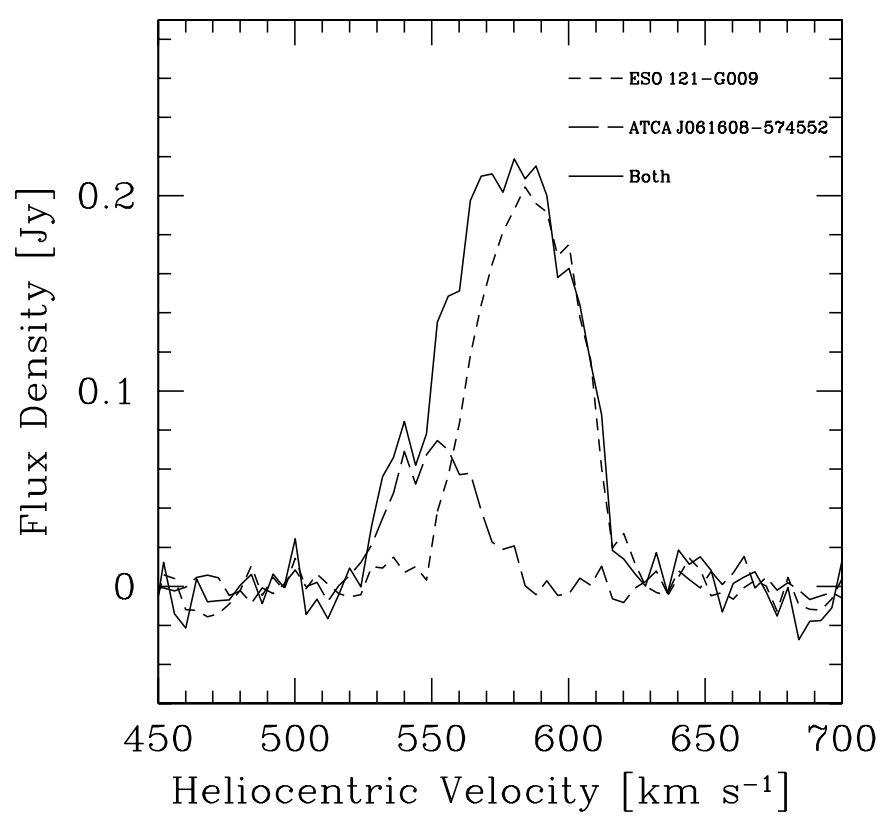

FIG. 6.-H I spectra of ESO 121-G020 and ATCA J061608-574552 as obtained from the ATCA, showing the spectra for both galaxies (dashed lines) and for the total system (solid line; as shown in Fig. 3c).

Huchtmeier (1992). The optical morphology of the galaxy is difficult to determine due to the high foreground star obscuration. We can only discern an elongated stellar feature (see Fig. $5 d$ ), which could be a galaxy disk. Alternatively, this could be a central bar, while the fainter spiral arms remain undetected. The $\mathrm{H}$ I distribution of ESO 428-G033 is nearly circular, indicating a disk that is close to face-on, which favors the optical feature being a bar rather than a disk. The position angle of velocity field is aligned with the optical emission.

\subsubsection{ESO 215-G?009}

This low surface brightness dwarf irregular galaxy was discussed in detail in Paper I, where we confirmed that it did have the unusually high $\mathcal{M}_{\mathrm{H}} / L_{B}$ value initially suggested by the combination of the BGC results and the magnitudes listed in LEDA. It is included here for comparison with the other galaxies, and we refer the reader to the previous work for more details on it. After obtaining new estimates of $m_{B}$ and $F_{\mathrm{H}_{\mathrm{I}}}$ for all our sample gal- axies, ESO 215-G?009 remains as the standout galaxy with $\mathcal{M}_{\mathrm{H}} / L_{B}=22 \pm 4 \mathcal{M}_{\odot} / L_{\odot, B}$. To our knowledge it has one of the highest $\mathrm{H}$ I mass-to-light ratios that has been confirmed by accurate measurement to date for any galaxy system, being approximately double the ratio of the best example in the literature, DDO 154 (Carignan \& Beaulieu 1989), and about 4 times the ratio of our next highest sample objects (ESO 572-G009 and ESO 428-G033). Like DDO 154 and another known high$\mathcal{M}_{\mathrm{H}} / L_{B}$ galaxy, NGC 3741 (Begum et al. 2005), ESO 215G?009 has a highly extended $\mathrm{H}$ I envelope, over 6 times the optical Holmberg radius. Begum et al. (2005) also point out that all three galaxies are isolated and have low tidal indexes from nearby galaxies. The nearest neighbor to ESO 215-G?009 that we can identify is approximately $1.7 \mathrm{Mpc}$ away, in the Centaurus A group.

\subsubsection{ESO 572-G009}

Although our H I flux density was in good agreement with the BGC and Fouqué et al. (1990), our apparent $B$ magnitude is about half a magnitude brighter than that given in LEDA and that of Lauberts \& Valentijn (1989). Despite this correction, ESO 572-G009 remains one of the few galaxies in our sample to retain a high $\mathcal{M}_{\mathrm{H}} / L_{B}$ value, at $4.8 \pm 1.1 \mathcal{M} \odot / L_{\odot, B}$. Morphologically it is a faint, low surface brightness galaxy and appears to have two stellar components, a brighter cigar-shaped central region (likely to be a central bar) surrounded by a fainter disk that extends only a short distance (see Fig. $5 f$ ).

\subsubsection{ESO 505-G007}

A previous measurement of the integrated $\mathrm{H}$ i flux density taken on the Effelsberg Radio Telescope by Richter \& Huchtmeier (1987), $19.3 \pm 2.2 \mathrm{Jy} \mathrm{km} \mathrm{s}^{-1}$, agrees with both the BGC and our ATCA results. However, literature $m_{B}$ measurements vary wildly (both $17.64 \pm 0.09$ and $15.95 \pm 0.09 \mathrm{mag}$ from Lauberts \& Valentijn [1989], depending on the isophotal level it is measured to). Our measurement is $\sim 3.5$ mag brighter than that listed in LEDA (corresponding to 25 times more luminous). The severe underestimate of the $B$-band apparent magnitude quoted by LEDA for this galaxy has a dramatic effect on the $\mathrm{H}$ I mass-tolight ratio. From the highest ratio of the $789 \mathrm{BGC}$ galaxies with $B$ magnitudes in LEDA, $\mathcal{M}_{\mathrm{H}_{\mathrm{I}}} / L_{B}$ for ESO 505-G007 has dropped down to a more typical ratio of $1.18 \pm 0.12 \mathcal{M}_{\odot} / L_{\odot, B}$, the lowest of our sample galaxies. We note that optically ESO 505G007 has an unusual irregular morphology (see Fig. $5 g$ ), with

TABLE 4

ATCA H i RESULTS

\begin{tabular}{|c|c|c|c|c|c|c|}
\hline $\begin{array}{l}\text { Name } \\
\text { (1) }\end{array}$ & $\begin{array}{c}\text { Beam (H I) } \\
(\operatorname{arcsec}) \\
(2)\end{array}$ & $\begin{array}{c}S_{\text {peak }} \\
\text { (Jy) } \\
(3)\end{array}$ & $\begin{array}{c}F_{\mathrm{H} \mathrm{I}_{\mathrm{I}}} \\
\left(\mathrm{Jy} \mathrm{km} \mathrm{^{-1 }}\right) \\
(4)\end{array}$ & $\begin{array}{c}v_{\text {sys }} \\
\left(\mathrm{km} \mathrm{s}^{-1}\right) \\
(5)\end{array}$ & $\begin{array}{c}w_{50} \\
\left(\mathrm{~km} \mathrm{~s}^{-1}\right) \\
(6)\end{array}$ & $\begin{array}{c}w_{20} \\
\left(\mathrm{~km} \mathrm{~s}^{-1}\right) \\
(7)\end{array}$ \\
\hline MCG -04-02-003 & $193 \times 129$ & $0.232 \pm 0.009$ & $16.2 \pm 0.6$ & $670 \pm 2$ & $112 \pm 2$ & $126 \pm 2$ \\
\hline 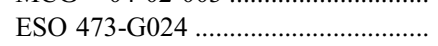 & $359 \times 205$ & $0.17 \pm 0.02$ & $5.7 \pm 0.9$ & $542 \pm 3$ & $37 \pm 2$ & $50 \pm 3$ \\
\hline 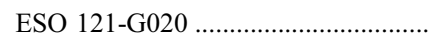 & $32 \times 30$ & $0.204 \pm 0.006$ & $9.1 \pm 0.3$ & $583 \pm 2$ & $47 \pm 2$ & $61 \pm 4$ \\
\hline ATCA J061608-574552 …................... & $32 \times 30$ & $0.075 \pm 0.006$ & $2.7 \pm 0.2$ & $554 \pm 4$ & $36 \pm 3$ & $56 \pm 8$ \\
\hline 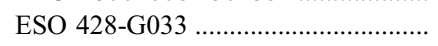 & $45 \times 30$ & $0.179 \pm 0.005$ & $14.5 \pm 0.3$ & $1728 \pm 2$ & $94 \pm 2$ & $110 \pm 2$ \\
\hline 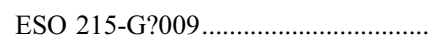 & $38 \times 35$ & $2.128 \pm 0.005$ & $122 \pm 4$ & $597 \pm 1$ & $64 \pm 2$ & $90 \pm 4$ \\
\hline 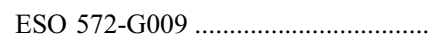 & $477 \times 327$ & $0.25 \pm 0.03$ & $7.2 \pm 1.3$ & $1740 \pm 4$ & $36 \pm 2$ & $49 \pm 2$ \\
\hline 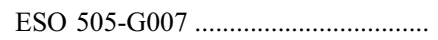 & $413 \times 304$ & $0.347 \pm 0.009$ & $21 \pm 3$ & $1776 \pm 2$ & $69 \pm 3$ & $88 \pm 5$ \\
\hline IC 4212 & $444 \times 357$ & $0.342 \pm 0.009$ & $46.0 \pm 1.0$ & $1476 \pm 1$ & $158 \pm 2$ & $172 \pm 2$ \\
\hline 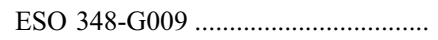 & $44 \times 27$ & $0.165 \pm 0.005$ & $13.1 \pm 0.3$ & $648 \pm 1$ & $86 \pm 2$ & $100 \pm 3$ \\
\hline
\end{tabular}

Notes.-Col. (1): Galaxy name (with ATCA J061608-574552 included). Col. (2): Size of the synthesized beam. Col. (3): H i peak flux density. Col. (4): Integrated H I flux density. Col. (5): H I systemic velocity from the $\mathrm{H}$ I line. Col. (6): Velocity width of the $\mathrm{H}$ I line at $50 \%$ of the peak flux density. Col. (7): Velocity width of the $\mathrm{H}$ I line at $20 \%$ of the peak flux density. 

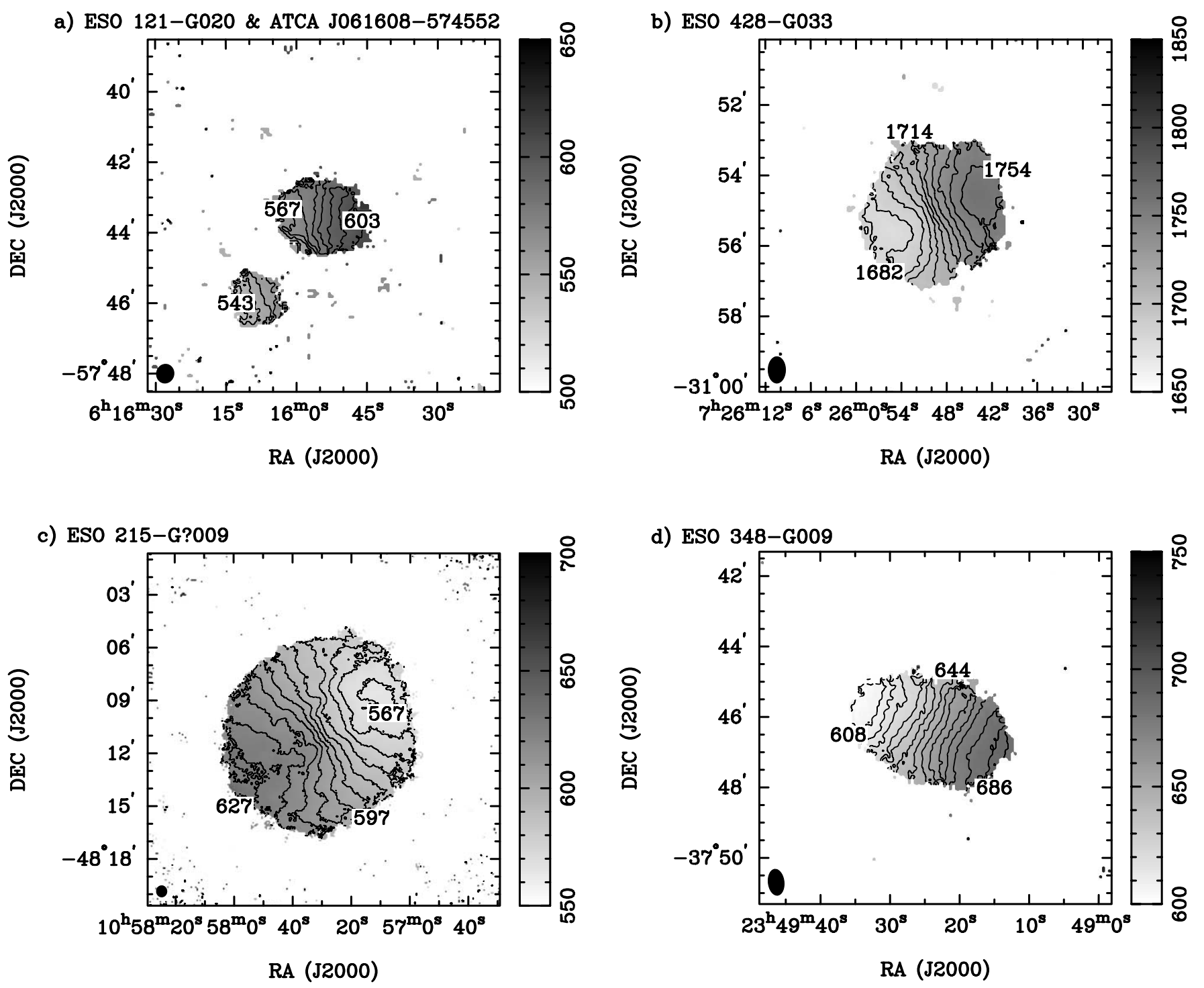

FIG. 7.- - H I velocity fields (moment 1 maps) for the four galaxy fields with the best resolution. The panels show (a) ESO 121-G020 and ATCA J061608574552, (b) ESO 428-G033, (c) ESO 215-G?009, and (d) ESO 348-G009. The numbers mark contour levels in kilometers per second.

several large clumpy structures and some ragged, spiral-arm-like features, which could suggest recent disruption and star formation triggered by interaction with ESO 505-G008.

$$
\text { 6.1.8. IC } 4212
$$

IC 4212 is an unusual galaxy to have in our sample since its optical morphology is that of a face-on spiral rather than the dwarf irregular we might expect for the magnitude given in LEDA. It has two bright, loosely wound arms, several fainter arms, and a small bright central bar (see Fig. 5h). The uncertainty in the apparent magnitude given in LEDA of $\pm 1.1 \mathrm{mag}$ is quite large. Our optical measurements find that IC 4212 is a much brighter galaxy than suggested by the $m_{B}$ value that LEDA lists (over $2 \mathrm{mag}$ ). This means that the $\mathcal{M}_{\mathrm{H}} / L_{B}$ value is not the

TABLE 5

Rotation Curve Fit for Galaxies with High-Resolution ATCA H i Data

\begin{tabular}{|c|c|c|c|c|c|}
\hline $\begin{array}{c}\text { Name } \\
(1)\end{array}$ & $\begin{array}{c}v_{\text {sys }} \\
\left(\mathrm{km} \mathrm{s}^{-1}\right) \\
(2)\end{array}$ & $\begin{array}{l}\text { P.A. } \\
\text { (deg) } \\
(3)\end{array}$ & $\begin{array}{c}i \\
(\mathrm{deg}) \\
(4)\end{array}$ & $\begin{array}{c}v_{\text {rot,max }} \\
\left(\mathrm{km} \mathrm{s}^{-1}\right) \\
(5)\end{array}$ & $\begin{array}{c}r_{\max } \\
(\operatorname{arcsec}) \\
(6)\end{array}$ \\
\hline 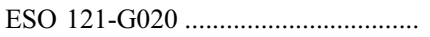 & $584.5 \pm 1.0$ & $262 \pm 2$ & $78 \pm 5$ & $21 \pm 2$ & $80 \pm 10$ \\
\hline 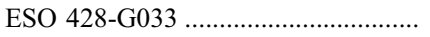 & $1717 \pm 3$ & $295 \pm 5$ & $11 \pm 5$ & $200_{-60}^{+160}$ & $130 \pm 5$ \\
\hline 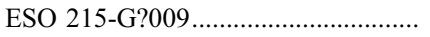 & $597 \pm 1$ & $119 \pm 2$ & $36 \pm 10$ & $51 \pm 8$ & $370 \pm 20$ \\
\hline 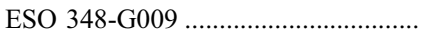 & $646 \pm 2$ & $245 \pm 3$ & $80 \pm 5$ & $50 \pm 5$ & $160 \pm 15$ \\
\hline
\end{tabular}

Notes.-Col. (1): Galaxy name. Col. (2): Systemic velocity. Col. (3): Position angle of the galaxy's receding side. Col. (4): Inclination angle. Col. (5): Maximum rotation velocity. Col. (6): Maximum radius. 


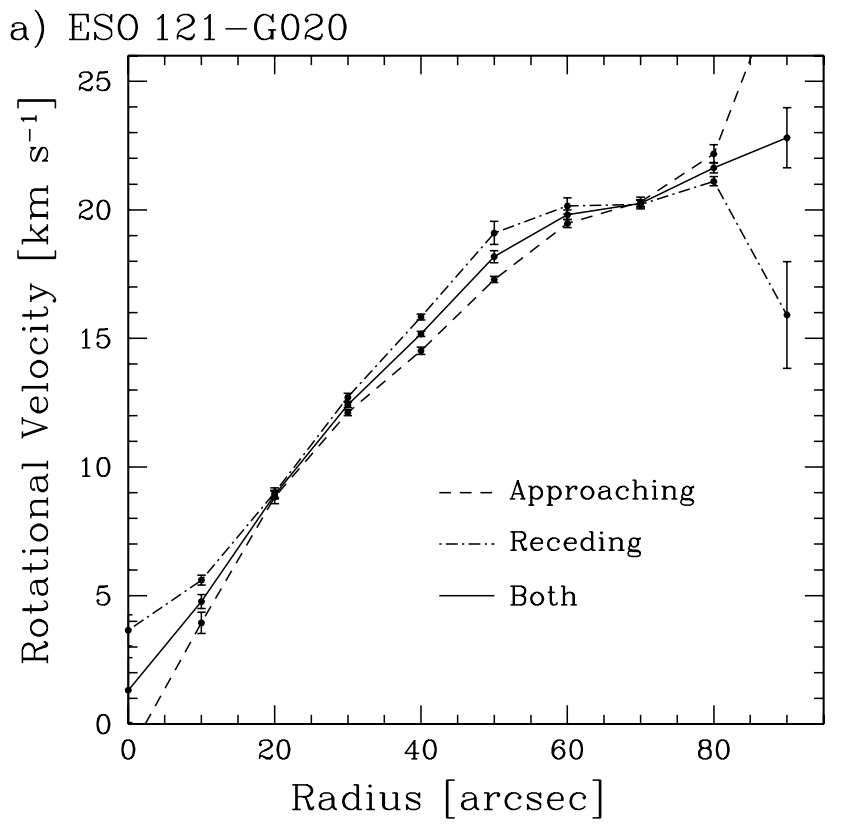

b) ESO 428-G033

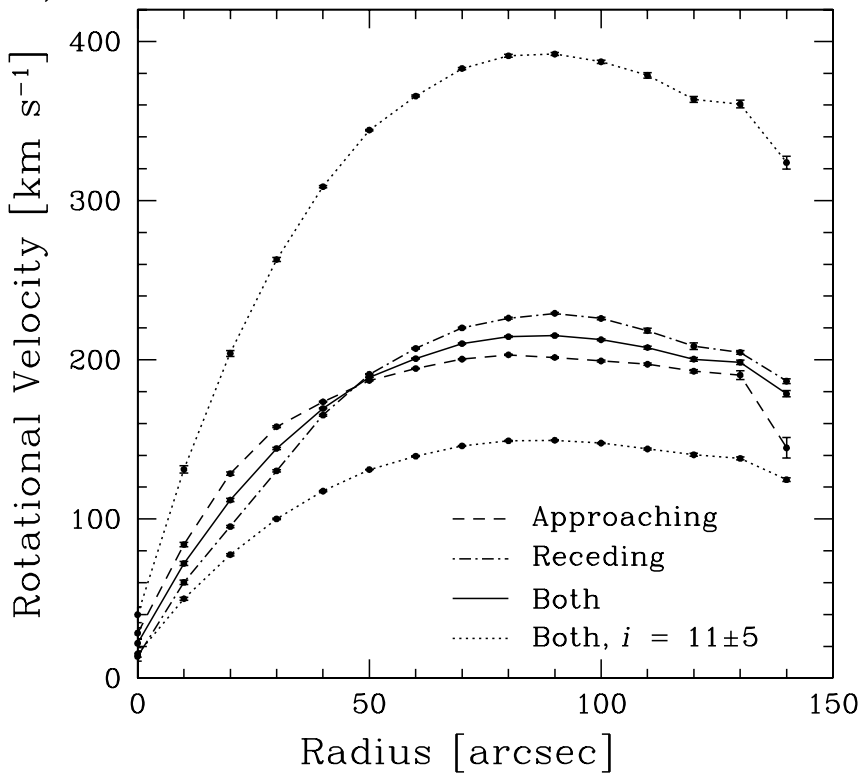

c) ESO 215-G?009

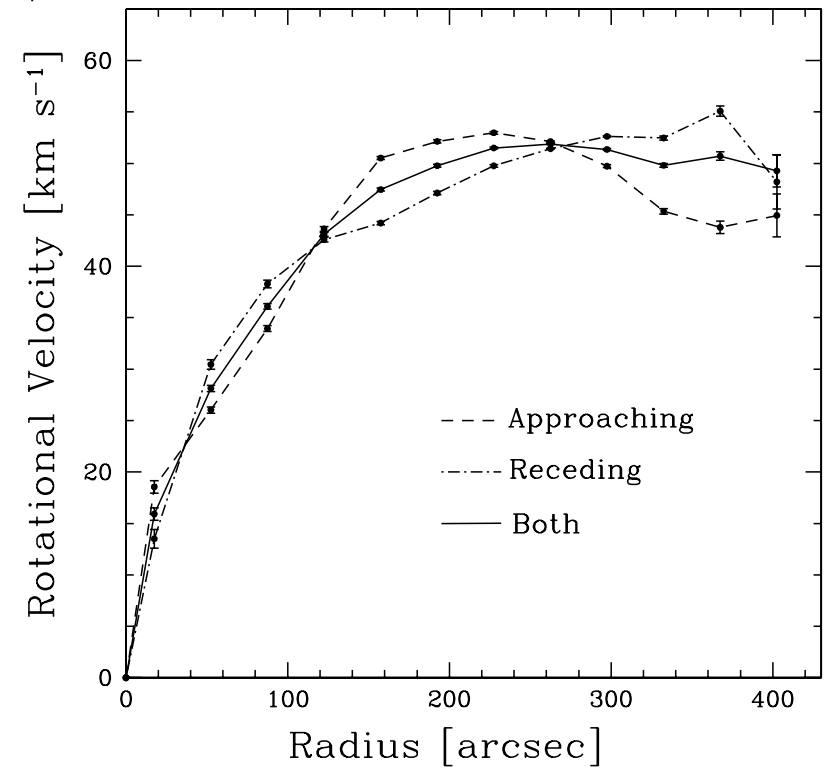

d) ESO 348-G009

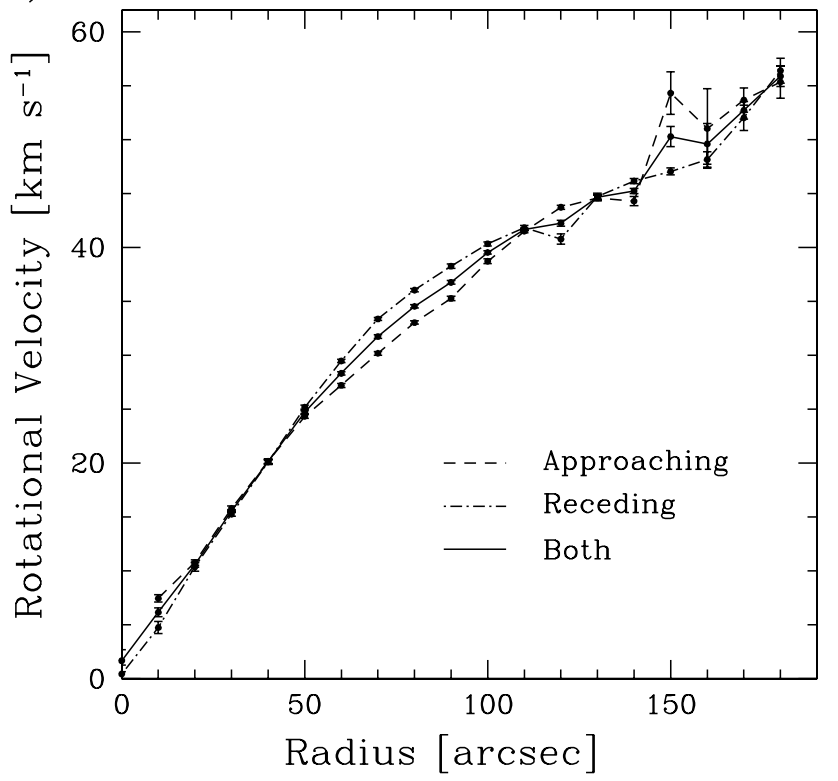

FIG. 8. - H I rotation curves as derived from the mean $\mathrm{H}$ I velocity field for the four sample galaxies to which they were fit. The panels show (a) ESO 121-G020, (b) ESO 428-G033, (c) ESO 215-G?009, and (d) ESO 348-G009. The three curves that are in all panels correspond to the approaching side (dashed line), receding side (dot-dashed line), and both sides (solid line). The dotted curves in the ESO 428-G033 panel show the difference that the inclination uncertainty makes for that galaxy.

extreme one initially suggested (the third highest ratio of the 789 BGC galaxies). However, IC 4212 retains a ratio of $2.55 \pm$ $0.17 \mathcal{M} \odot / L_{\odot, B}$, which is relatively high for a galaxy with such a distinct spiral structure, typical values for late-type spirals being less than $1 \mathcal{M} \odot / L_{\odot, B}$ (Roberts \& Haynes 1994). We measure a deconvolved $\mathrm{H}$ I diameter of $\sim 200^{\prime \prime}$, suggesting it may extend significantly beyond the optical disk.

\subsubsection{ESO 348-G009}

The apparent $B$ magnitude we measured for ESO 348-G009 was again brighter than the value listed in LEDA, while the $\mathrm{H}_{\mathrm{I}}$ flux density is consistent with the BGC result. This means we end up with an $\mathrm{H}$ I mass-to-light ratio of $1.56 \pm 0.16 \mathcal{M}_{\odot} / L_{\odot, B}$, down from the preliminary value of $9 \pm 7 \mathcal{M} \odot / L_{\odot, B}$. The optical image shows an edge-on disk galaxy extending east-west, with some clumpy structures visible along the length of the disk (see Fig. 5i). The position angle of the $\mathrm{H}$ I velocity field is aligned closely with the stellar disk.

\subsection{Distance Uncertainties}

As was discussed in Paper I, the use of distances calculated from Local Group velocities for galaxies in the local universe can be problematic, as the peculiar velocities in nearby groups are potentially of similar order to the redshifts themselves and the local Hubble flow can differ from the cosmological expansion. Evidence from studies on the Sculptor group (Jerjen et al. 
TABLE 6

2.3 m Telescope Optical Results

\begin{tabular}{|c|c|c|c|c|c|c|c|}
\hline $\begin{array}{c}\text { Name } \\
\text { (1) }\end{array}$ & $\begin{array}{c}\text { Band } \\
\text { (2) }\end{array}$ & $\begin{array}{c}m_{T}^{\mathrm{a}} \\
(\mathrm{mag}) \\
(3)\end{array}$ & $\begin{array}{c}\mu_{0}^{\mathrm{a}} \\
\left(\mathrm{mag}^{\left.\operatorname{arcsec}^{-2}\right)}\right. \\
(4)\end{array}$ & $\begin{array}{c}\langle\mu\rangle_{\mathrm{eff}^{\mathrm{a}}} \\
\left(\mathrm{mag}^{\left.\operatorname{arcsec}^{-2}\right)}\right. \\
(5)\end{array}$ & $\begin{array}{c}r_{\mathrm{eff}} \\
(\operatorname{arcsec}) \\
(6)\end{array}$ & $\begin{array}{c}r_{\mathrm{H}, 0} \\
(\operatorname{arcsec}) \\
(7)\end{array}$ & $\begin{array}{c}A_{G} \\
(\mathrm{mag}) \\
(8)\end{array}$ \\
\hline \multirow{4}{*}{ MCG -04-02-003 } & $B$ & $15.32 \pm 0.06$ & $22.34 \pm 0.02$ & $23.77 \pm 0.04$ & $19.6 \pm 0.7$ & $51.0 \pm 2.0$ & $0.08 \pm 0.01$ \\
\hline & V & $14.91 \pm 0.05$ & $21.89 \pm 0.01$ & $22.98 \pm 0.04$ & $16.4 \pm 0.6$ & $\ldots$ & $0.06 \pm 0.01$ \\
\hline & $R$ & $14.40 \pm 0.05$ & $21.60 \pm 0.01$ & $22.68 \pm 0.05$ & $18.1 \pm 0.7$ & $\ldots$ & $0.05 \pm 0.01$ \\
\hline & $I$ & $13.60 \pm 0.06$ & $21.17 \pm 0.01$ & $22.76 \pm 0.05$ & $27.1 \pm 1.0$ & $\ldots$ & $0.04 \pm 0.01$ \\
\hline \multirow[t]{4}{*}{ 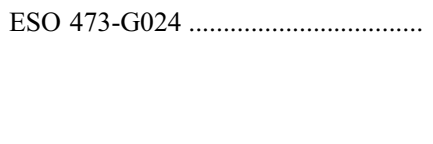 } & $B$ & $16.38 \pm 0.06$ & $24.63 \pm 0.04$ & $25.30 \pm 0.04$ & $24.3 \pm 0.8$ & $32.4 \pm 0.8$ & $0.08 \pm 0.01$ \\
\hline & $V$ & $15.44 \pm 0.07$ & $23.73 \pm 0.03$ & $24.46 \pm 0.03$ & $25.4 \pm 0.7$ & $\ldots$ & $0.06 \pm 0.01$ \\
\hline & $R$ & $15.19 \pm 0.03$ & $23.51 \pm 0.03$ & $24.18 \pm 0.02$ & $25.1 \pm 0.4$ & $\ldots$ & $0.05 \pm 0.01$ \\
\hline & $I$ & $14.76 \pm 0.07$ & $23.42 \pm 0.08$ & $24.35 \pm 0.02$ & $33.0 \pm 0.9$ & $\ldots$ & $0.04 \pm 0.01$ \\
\hline \multirow{2}{*}{ 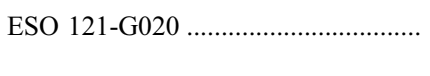 } & $B$ & $15.27 \pm 0.05$ & $23.36 \pm 0.03$ & $23.95 \pm 0.02$ & $21.7 \pm 0.4$ & $47.0 \pm 2.0$ & $0.17 \pm 0.03$ \\
\hline & $R$ & $14.62 \pm 0.04$ & $22.71 \pm 0.03$ & $23.29 \pm 0.02$ & $21.6 \pm 0.5$ & $\ldots$ & $0.11 \pm 0.02$ \\
\hline \multirow[t]{2}{*}{ ATCA J061608-574552. } & $B$ & $17.01 \pm 0.06$ & $22.81 \pm 0.04$ & $23.34 \pm 0.02$ & $7.4 \pm 0.3$ & $18.6 \pm 0.6$ & $0.17 \pm 0.03$ \\
\hline & $R$ & $16.36 \pm 0.06$ & $22.07 \pm 0.02$ & $22.72 \pm 0.02$ & $7.5 \pm 0.2$ & $\ldots$ & $0.11 \pm 0.02$ \\
\hline \multirow{4}{*}{ ESO 428-G033 ………....................... } & $B$ & $16.90 \pm 0.10$ & $23.33 \pm 0.11$ & $24.69 \pm 0.02$ & $14.4 \pm 0.5$ & $37.2 \pm 1.2$ & $1.10 \pm 0.18$ \\
\hline & $V$ & $16.13 \pm 0.10$ & $23.12 \pm 0.02$ & $24.23 \pm 0.05$ & $16.7 \pm 0.8$ & $\ldots$ & $0.85 \pm 0.14$ \\
\hline & $R$ & $15.61 \pm 0.08$ & $22.56 \pm 0.02$ & $23.48 \pm 0.05$ & $15.0 \pm 0.9$ & $\ldots$ & $0.68 \pm 0.11$ \\
\hline & $I$ & $15.04 \pm 0.09$ & $22.01 \pm 0.03$ & $22.95 \pm 0.04$ & $15.2 \pm 0.7$ & $\ldots$ & $0.50 \pm 0.08$ \\
\hline \multirow[t]{4}{*}{ 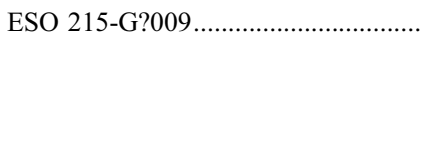 } & $B$ & $16.13 \pm 0.07$ & $24.97 \pm 0.03$ & $25.48 \pm 0.02$ & $29.7 \pm 0.6$ & $57.6 \pm 0.6$ & $0.95 \pm 0.15$ \\
\hline & $V$ & $14.89 \pm 0.06$ & $23.65 \pm 0.03$ & $24.14 \pm 0.02$ & $28.3 \pm 0.7$ & $\ldots$ & $0.73 \pm 0.12$ \\
\hline & $R$ & $14.38 \pm 0.05$ & $23.16 \pm 0.02$ & $23.64 \pm 0.03$ & $28.4 \pm 0.5$ & $\ldots$ & $0.59 \pm 0.09$ \\
\hline & $I$ & $13.76 \pm 0.06$ & $22.91 \pm 0.04$ & $23.40 \pm 0.03$ & $33.9 \pm 0.8$ & $\ldots$ & $0.43 \pm 0.07$ \\
\hline \multirow{3}{*}{ 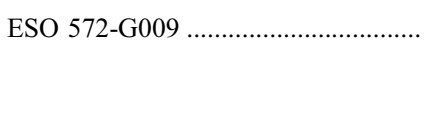 } & $B$ & $16.79 \pm 0.05$ & $24.96 \pm 0.05$ & $26.03 \pm 0.02$ & $28.1 \pm 0.7$ & $30.6 \pm 1.2$ & $0.16 \pm 0.03$ \\
\hline & $V$ & $15.65 \pm 0.07$ & $23.69 \pm 0.02$ & $25.12 \pm 0.02$ & $31.1 \pm 0.8$ & $\ldots$ & $0.12 \pm 0.02$ \\
\hline & $R$ & $15.40 \pm 0.04$ & $23.42 \pm 0.03$ & $24.75 \pm 0.01$ & $29.6 \pm 0.5$ & $\ldots$ & $0.10 \pm 0.02$ \\
\hline \multirow[t]{3}{*}{ 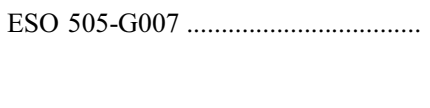 } & $B$ & $14.20 \pm 0.06$ & $23.99 \pm 0.04$ & $24.00 \pm 0.01$ & $36.4 \pm 0.7$ & $82.8 \pm 1.2$ & $0.36 \pm 0.06$ \\
\hline & $V$ & $14.48 \pm 0.05$ & $23.89 \pm 0.04$ & $23.96 \pm 0.01$ & $31.3 \pm 0.6$ & $\ldots$ & $0.28 \pm 0.04$ \\
\hline & $R$ & $13.97 \pm 0.04$ & $23.46 \pm 0.04$ & $23.66 \pm 0.02$ & $34.6 \pm 0.5$ & $\ldots$ & $0.22 \pm 0.04$ \\
\hline \multirow[t]{3}{*}{ IC 4212} & $B$ & $14.11 \pm 0.04$ & $22.38 \pm 0.02$ & $24.42 \pm 0.02$ & $46.0 \pm 1.0$ & $98.4 \pm 0.6$ & $0.19 \pm 0.03$ \\
\hline & $V$ & $13.69 \pm 0.05$ & $22.00 \pm 0.03$ & $23.98 \pm 0.01$ & $45.7 \pm 0.9$ & $\ldots$ & $0.14 \pm 0.02$ \\
\hline & $R$ & $13.29 \pm 0.06$ & $21.53 \pm 0.02$ & $23.53 \pm 0.02$ & $44.5 \pm 0.9$ & $\ldots$ & $0.12 \pm 0.02$ \\
\hline \multirow[t]{4}{*}{ 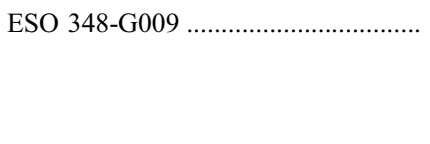 } & $B$ & $14.81 \pm 0.07$ & $23.78 \pm 0.04$ & $24.79 \pm 0.03$ & $39.5 \pm 0.9$ & $72.0 \pm 0.6$ & $0.06 \pm 0.01$ \\
\hline & V & $14.68 \pm 0.06$ & $23.42 \pm 0.03$ & $24.40 \pm 0.03$ & $35.1 \pm 1.0$ & $\ldots$ & $0.04 \pm 0.01$ \\
\hline & $R$ & $14.48 \pm 0.05$ & $23.13 \pm 0.03$ & $24.03 \pm 0.03$ & $32.4 \pm 0.8$ & $\ldots$ & $0.04 \pm 0.01$ \\
\hline & $I$ & $13.75 \pm 0.10$ & $22.75 \pm 0.03$ & $23.72 \pm 0.02$ & $39.3 \pm 1.1$ & $\ldots$ & $0.03 \pm 0.01$ \\
\hline
\end{tabular}

Notes.-Col. (1): Galaxy name. Col. (2): Broadband (Cousins) filter used. Col. (3): Total apparent magnitude. Col. (4): Central surface brightness. Col. (5): Effective surface brightness (i.e., the average surface brightness out to the half-light radius). Col. (6): Half-light (effective) radius. Col. (7): Radius out to $\mu=26.6$ mag $\operatorname{arcsec}^{-2}$ (i.e., the Holmberg radius in the $B$ band, extinction corrected). Col. (8): Galactic extinction correction from SFD98. No Galactic extinction correction was applied to the apparent magnitude and surface brightness.

${ }^{\text {a }}$ Correction for Galactic extinction not applied.

1998) suggests that the local velocity-distance relationship is much steeper in the direction of this group than for galaxies farther out due to the probable gravitational influence of the Local Group. If we use the Hubble constant of Jerjen et al. (1998), $H_{0}=119 \mathrm{~km} \mathrm{~s}^{-1} \mathrm{Mpc}^{-1}$, for the three Sculptor group members in our sample (membership confirmed using Côté et al. 1997), then the Local Group velocity distances to these galaxies would be less than listed in Table 1, with 6.0 Mpc for MCG -04-02003, 4.8 Mpc for ESO 473-G024, and 5.3 Mpc for ESO 348G009. This would put all three on the far side of the Sculptor group (Jerjen et al. 1998) and would mean that the distance-dependent quantities in Table 7 (everything except $\mathcal{M}_{\mathrm{H}} / L_{B}$ ) would require adjustment.

\section{DISCUSSION}

\subsection{The Revised H I Mass-to-Light Ratios}

The plot in Figure 11 shows $\mathcal{M}_{\mathrm{H}_{\mathrm{I}}} / L_{B}$ versus $M_{B}$ (as in Fig. 1) with the new positions of the nine target galaxies resulting from our observations. The lines connect our results (large points with error bars) to the initial estimates (open circles). While the new measurements resulted in lower $\mathcal{M}_{\mathrm{H}_{\mathrm{I}}} / L_{B}$ values for all selected galaxies, the decrease is particularly significant for galaxies that had preliminary values of $\mathcal{M}_{\mathrm{H}_{\mathrm{I}}} / L_{B}>5 \mathcal{M}_{\odot} / L_{\odot, B}$. While disappointing, our result is not too surprising given that we selected galaxies from the BGC with initially the most extreme $\mathcal{M}_{\mathrm{H}} / L_{B}$ values and large uncertainties in their optical magnitudes, so we preferentially selected galaxies with underestimated $m_{B}$. For many of our target galaxies optical magnitudes exist that agree with our results (e.g., Lauberts \& Valentijn 1989), but the mean magnitudes available from LEDA were generally highly underestimated.

All but one of the selected galaxies, ESO 215-G?009, now have revised $\mathrm{H}$ I mass-to-light ratios in the range $\sim 1-5$ $\mathcal{M}_{\odot} / L_{\odot, B}$. Such revisions are by no means uncommon among claims of high- $\mathcal{M}_{\mathrm{H}} / L_{B}$ galaxies, as we discussed in $\S 6.2$ of Paper I and as seen in van Zee et al. (1997) and Chung et al. (2002). The faint luminosity of these galaxies and the difficulty in getting high-quality data in both the optical and radio regimes make examples of high- $\mathcal{M}_{\mathrm{H}_{\mathrm{I}}} / L_{B}$ galaxies difficult to find. Only a few other galaxies with confirmed high $\mathcal{M}_{\mathrm{H}} / L_{B}$ values are in the literature, most notably DDO $154\left(9.4 \mathcal{M}_{\odot} / L_{\odot, B}\right.$; 

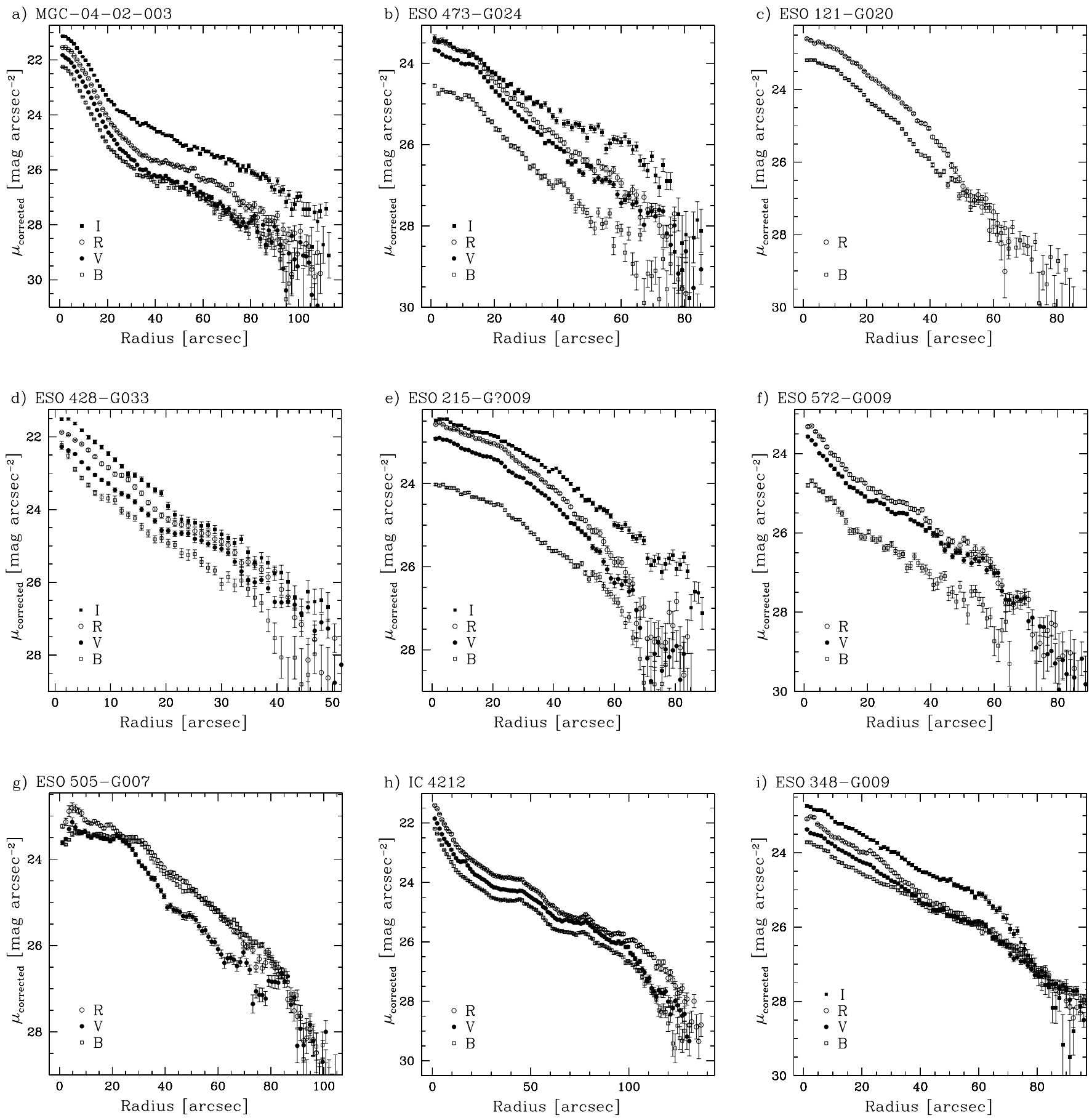

FIG. 9. - BVRI surface brightness profiles of the nine galaxies. The galaxies shown in the respective panels are as in Fig. 2. All profiles were corrected for Galactic extinction (see Table 6).

Carignan \& Beaulieu 1989; Hoffman et al. 1993), UGCA 292 $\left(7.0 \mathcal{M}_{\odot} / L_{\odot, B}\right.$; Young et al. 2003), and NGC $3741\left(5.8 \mathcal{M}_{\odot} / L_{\odot, B}\right.$; Begum et al. 2005).

While we must be careful of small-number statistics, the trend of all the findings of current studies strongly suggests that there do not appear to be large numbers of "dim" galaxies like ESO $215-\mathrm{G}$ ?009 in the local universe. Therefore, high- $\mathcal{M}_{\mathrm{H}} / L_{B}$ galaxies cannot account for much of the discrepancy between observations and theoretical predictions of low-mass galaxy numbers. But there are other possible ways that galaxies could be missed observationally and several suggestions of how they might be detected. Some methods have been proposed for finding "empty" dark matter halos, such as the suggestion to use Milky Way halo microlensing statistics to look for dark matter satellite influence (Widrow \& Dubinski 1998), or to analyze the gravitational lensing of quasars to determine the dark matter subhalos of the lensing object (Moore et al. 1999; Dalal \& Kochanek 2002). However, the existence of halos without baryons is still highly speculative.

True "dark" galaxies in the form of isolated, rotating, extragalactic $\mathrm{H}$ i clouds have so far proven elusive (Ryan-Weber et al. 2002; Koribalski et al. 2004; Doyle et al. 2005). Some isolated H I sources have been found in HIPASS (Kilborn et al. 2000; Ryder et al. 2001; Ryder \& Koribalski 2004) and were interpreted as 


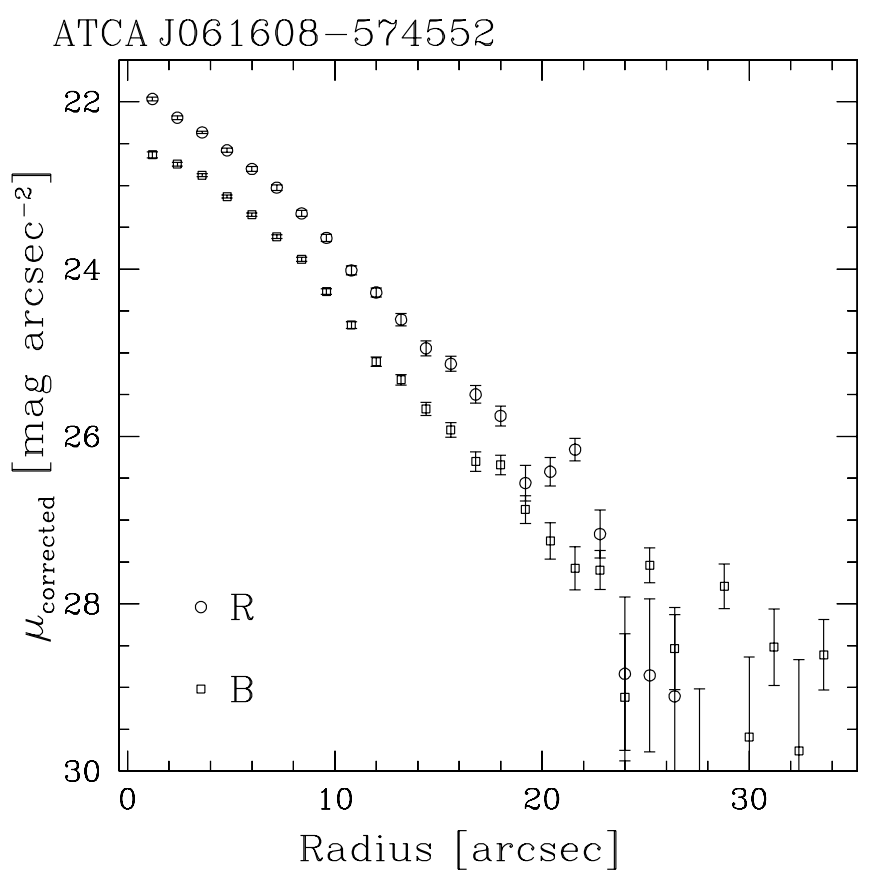

FIG. 10. - BR surface brightness profiles for ATCA J061608-574552. Both profiles were corrected for Galactic extinction (see Table 6).

high-velocity clouds or tidal debris by the respective authors. A "dark galaxy" that was recently claimed to be close to the onearmed spiral NGC 4254, in the outskirts of the Virgo Cluster (Minchin et al. 2005), also appears to be tidal debris (Bekki et al. 2005; see also Oosterloo \& van Gorkom 2005). Taylor \& Webster (2005) have discussed theoretically that an isolated $\mathrm{H}$ I cloud that formed without a stellar component is likely to be unstable to star formation and therefore would not remain dark.

\subsection{The Importance of Dust Extinction}

As well as the accuracy of the $B$-band photometry and $\mathrm{H}$ I flux density, it is also important to discuss the one contributor to $\mathcal{M}_{\mathrm{H}_{\mathrm{I}}} / L_{B}$ that is beyond the scope of our observations, the dust extinction due to both our Galaxy (see Table 6) and the host galaxy ("internal" extinction). As we noted in Paper I, Galactic extinction is particularly important for ESO 215-G?009 due to

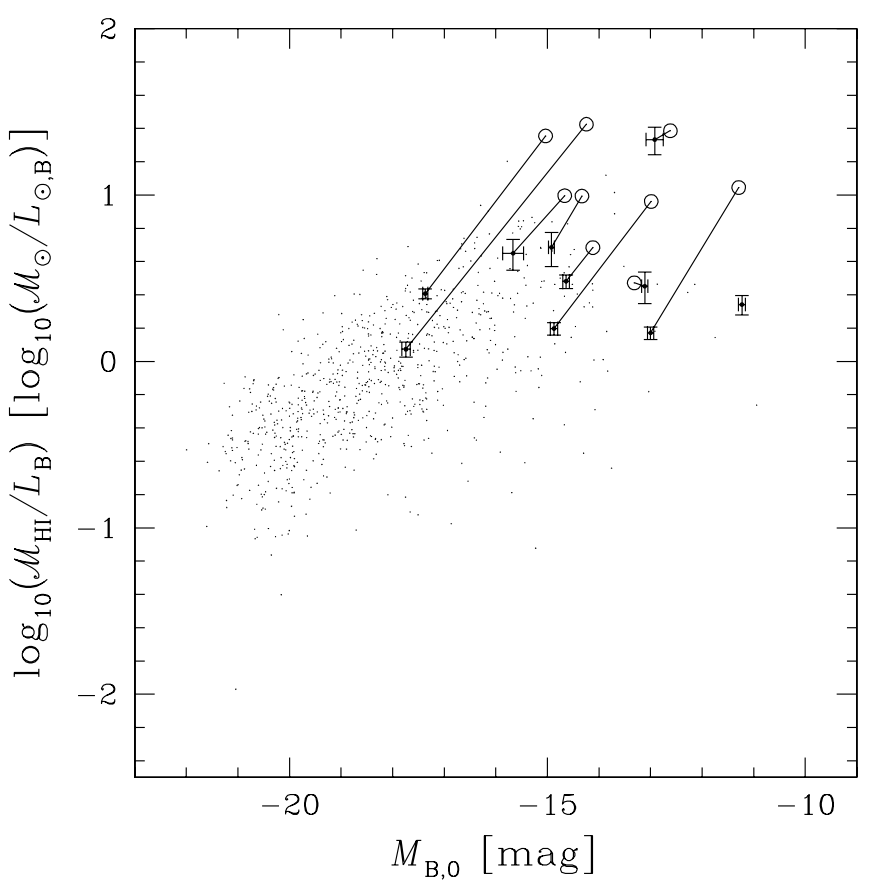

FIG. 11.-H I mass-to-light ratio vs. absolute $B$ magnitude, with the 789 galaxies shown in Fig. 1 marked with small points. The nine galaxies in this paper are shown with open circles (preliminary values as in Fig. 1) and large points with error bars (revised values as derived in this paper). Lines link the preliminary to the revised values, and the point by itself is ATCA J061608-574552.

its sky position only $10^{\circ} .5$ from the Galactic plane. ESO 428G033 at $b=-6^{\circ} .9$ is the only other galaxy that is subject to similar Galactic extinction $\left(A_{B}=1.10 \pm 0.18\right.$ mag; Schlegel et al. 1998, hereafter SFD98).

The uncertainties in the SFD98 Galactic extinction grow proportionally to the value. This means that it only contributes a significant fraction to the total error in $\mathcal{M}_{\mathrm{H}_{\mathrm{I}}} / L_{B}$ for ESO 215 G?009 and ESO 428-G033. Close to the Galactic plane the dust distribution can be patchy. Figure 12 shows the SFD98 dust extinction maps in terms of $A_{B}$ for the regions around ESO 428G033 and ESO 215-G?009, with the last H i contour from Figure 2 superposed for reference. Both maps show relatively low variation in the amount of extinction over the field around the galaxy, less than the uncertainty in $A_{B}$ in both cases. This suggests that the SFD98 value at the position of both galaxies is an

TABLE 7

Summary of Derived Galaxy Properties from ATCA and $2.3 \mathrm{~m}$ Data

\begin{tabular}{|c|c|c|c|c|c|c|c|}
\hline $\begin{array}{l}\text { Name } \\
\text { (1) }\end{array}$ & $\begin{array}{c}M_{B} \\
(\mathrm{mag}) \\
(2)\end{array}$ & $\begin{array}{c}L_{B} \\
\left(\times 10^{7} L_{\odot, B}\right) \\
(3)\end{array}$ & $\begin{array}{c}\mathcal{M}_{\mathrm{H}_{\mathrm{I}}} \\
\left(\times 10^{7} \mathcal{M}_{\odot}\right) \\
(4)\end{array}$ & $\begin{array}{c}\mathcal{M}_{\mathrm{H}_{\mathrm{I}}} / L_{B} \\
\left(\mathcal{M}_{\odot} / L_{\odot, B}\right) \\
(5)\end{array}$ & $\begin{array}{c}\mathcal{M}_{\text {tot }} \\
\left(\times 10^{9} \mathcal{M}_{\odot}\right) \\
(6)\end{array}$ & $\begin{array}{c}\mathcal{M}_{\mathrm{H}_{\mathrm{I}}} / \mathcal{M}_{\text {tot }} \\
(7)\end{array}$ & $\begin{array}{c}\mathcal{M}_{\text {tot }} / L_{B} \\
\left(\mathcal{M}_{\odot} / L_{\odot, B}\right) \\
(8)\end{array}$ \\
\hline MCG -04-02-003. & $-14.65 \pm 0.06$ & $11.3 \pm 0.6$ & $34.5 \pm 1.3$ & $3.0 \pm 0.3$ & ... & $\cdots$ & $\ldots$ \\
\hline ESO 473-G024 . & $-13.10 \pm 0.06$ & $2.7 \pm 0.1$ & $7.8 \pm 1.2$ & $2.8 \pm 0.6$ & $\ldots$ & $\cdots$ & $\cdots$ \\
\hline ESO $121-G 020 \ldots .$. & $-12.97 \pm 0.06$ & $2.39 \pm 0.13$ & $3.61 \pm 0.12$ & $1.50 \pm 0.13$ & $0.16 \pm 0.05$ & $0.23 \pm 0.11$ & $7 \pm 4$ \\
\hline ATCA J061608-574552.. & $-11.23 \pm 0.07$ & $0.48 \pm 0.03$ & $1.07 \pm 0.08$ & $2.2 \pm 0.3$ & $\ldots$ & $\ldots$ & $\ldots$ \\
\hline 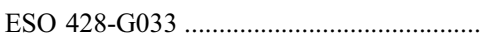 & $-16.7 \pm 0.2$ & $28 \pm 5$ & $130 \pm 3$ & $4.5 \pm 0.9$ & $110_{-70}^{+190}$ & $0.012_{-0.008}^{+0.020}$ & $\sim 380$ \\
\hline ESO $215-G ? 009 \ldots$ & $-12.9 \pm 0.2$ & $2.3 \pm 0.4$ & $50.8 \pm 1.7$ & $22 \pm 4$ & $4.5 \pm 1.6$ & $0.11 \pm 0.04$ & $200 \pm 110$ \\
\hline ESO 572-G009 .... & $-14.92 \pm 0.06$ & $14.5 \pm 0.8$ & $71 \pm 13$ & $4.8 \pm 1.1$ & $\ldots$ & $\ldots$ & $\ldots$ \\
\hline ESO 505-G007 ..... & $-17.75 \pm 0.08$ & $196 \pm 14$ & $210 \pm 30$ & $1.1 \pm 0.2$ & $\ldots$ & $\ldots$ & $\cdots$ \\
\hline IC $4212 \ldots \ldots \ldots \ldots \ldots$ & $-17.36 \pm 0.05$ & $137 \pm 6$ & $356 \pm 8$ & $2.55 \pm 0.17$ & $\ldots$ & $\ldots$ & $\ldots$ \\
\hline 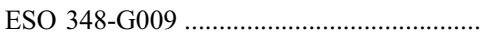 & $-14.87 \pm 0.07$ & $13.8 \pm 0.9$ & $21.8 \pm 0.5$ & $1.56 \pm 0.16$ & $3.8 \pm 1.1$ & $0.06 \pm 0.02$ & $27 \pm 10$ \\
\hline
\end{tabular}

Notes.-Col. (1): Galaxy name. Col. (2): B-band absolute magnitude. Col. (3): B-band luminosity. Col. (4): H I mass. Col. (5): H I mass-to- $B$-band luminosity ratio. Col. (6): Total dynamical mass. Col. (7): $\mathrm{H}$ I mass-to-total mass ratio. Col. (8): Total mass-to- $B$-band luminosity ratio. 
ESO 428-G033

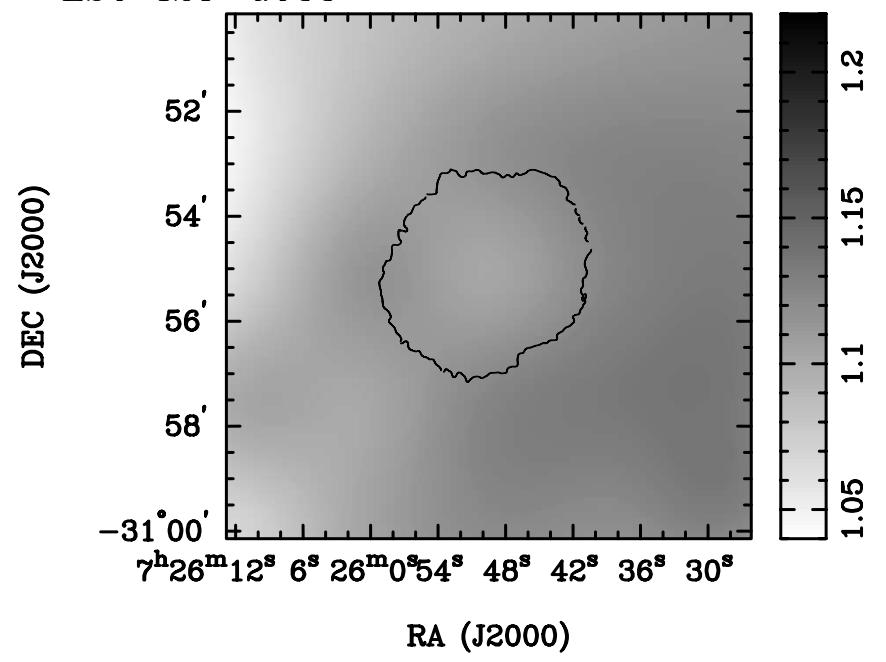

\section{ESO 215-G?009}

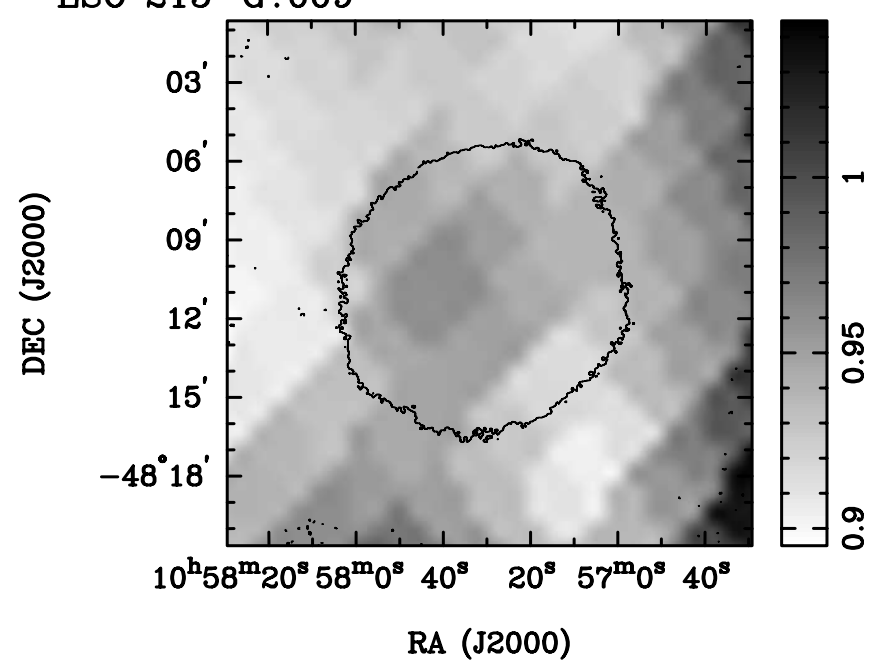

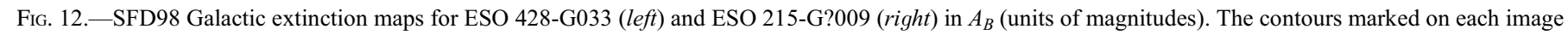
correspond to the lowest $\mathrm{H}$ I contours for each galaxy as shown in Fig. 2.

accurate representation of the true Galactic extinction. ESO 428G033 was studied by Kraan-Korteweg \& Huchtmeier (1992) as part of an investigation of galaxies in a region of the Galactic plane with reduced dust extinction. Their results are in agreement with our measurements, and in general they found that the properties of their sample objects were typical of other samples of nearby galaxies despite the relatively high extinction. It is worth noting that even if the Galactic extinction was much higher at the position of ESO 215-G?009, say by $0.5 \mathrm{mag}$, the galaxy would still have a very high $\mathcal{M}_{\mathrm{H}_{\mathrm{I}}} / L_{B}$ value of $14 \pm$ $4 \mathcal{M} \odot / L_{\odot, B}$.

While we can at least get a relatively accurate estimate of Galactic extinction, dust extinction internal to the host galaxy itself is much harder to quantify. Dust extinction in late-type galaxies is poorly understood but thought to be lower than in early-type spirals due to low metallicity and ineffective dust accretion processes (Dwek 1998; Hirashita 1999). We would expect a disk galaxy to have significant extinction when viewed close to edge-on, as with ESO 348-G009 ( $i=80^{\circ} \pm 5^{\circ}$; see Table 5), due to the geometry of the dust distribution. We discussed ESO 215-G?009's possible internal extinction in Paper I, concluding that it is most likely low for this close-to-face-on galaxy. The galaxy ESO 121-G020 may have a high inclination angle of $78^{\circ} \pm 5^{\circ}$ (Table 5), in contrast to the optical dimensions, and a moderate amount of internal extinction. The optical emission of the galaxy ESO 428-G033 is highly obscured by foreground stars. Due to the current inability to estimate internal extinction, we have not accounted for it in our $\mathcal{M}_{\mathrm{H}} / L_{B}$ calculations.

\subsection{Physical Characteristics of High- $\mathcal{M}_{\mathrm{H}_{\mathrm{I}}} / L_{B}$ Galaxies and Implications for Their Existence}

Several common elements between the galaxies with the most extreme $\mathcal{M}_{\mathrm{H}_{\mathrm{I}}} / L_{B}$ values are becoming more evident. The three best examples, ESO 215-G?009 $\left(22 \pm 4 \mathcal{M}_{\odot} / L_{\odot, B}\right.$; Paper I $)$, DDO $154\left(9.4 \mathcal{M}_{\odot} / L_{\odot, B}\right.$; Carignan \& Beaulieu 1989; Hoffman et al. 1993), and NGC 3741 (5.8 $\mathcal{M} \odot / L_{\odot, B}$; Begum et al. 2005), all have $\mathrm{H}$ I envelopes that are 5-8 times the optical Holmberg radius, as IC 4212 may also have. This may be because the gas is at a low density and is in a stable state, as was seen for ESO 215 G?009 in Paper I. All these galaxies also have low tidal indexes (Karachentsev et al. 2004), indicating that they are isolated in space and have little external stimulation to form stars. Despite having very low stellar content for their dynamical masses, the baryonic masses of the galaxies are always of the order of $\sim 10 \%$ of the total dynamical mass, which is a typical fraction seen in galaxies from $L_{*}$ to the dwarf regime (Begum et al. 2005). This suggests that high- $\mathcal{M}_{\mathrm{H}_{\mathrm{I}}} / L_{B}$ galaxies are not lacking the baryons to create stars, but are underluminous as they lack either the internal or external stimulation for further star formation.

Skillman et al. (2003b) obtained spectra of $\mathrm{H}$ II regions within five Sculptor group galaxies, including two in our sample, ESO 473-G024 and ESO 348-G009. For ESO 473-G024, for which we found a moderately high $\mathcal{M}_{\mathrm{H}_{\mathrm{I}}} / L_{B}$ value, they were able to produce oxygen and nitrogen abundances. The oxygen abundance indicated a low metallicity that is typical of other late-type galaxies. Normally, dwarf galaxies with a similar low metallicity have low nitrogen-to-oxygen ratios in a narrow range around an average of $\log (\mathrm{N} / \mathrm{O}) \simeq-1.6$ (Izotov \& Thuan 1999). It is thought that this is because these galaxies are undergoing their first burst of star formation and the nitrogen from this burst has not yet had time to dissipate into the interstellar medium (coming from Type II supernovae of intermediate-mass stars, while oxygen comes from higher mass stars; see Skillman et al. 2003b). However, Skillman et al. (2003b) found that the N/O ratio for ESO 473-G024 was relatively high. Most importantly for our study, they compared these results to a study of the high- $\mathcal{M}_{\mathrm{H}_{\mathrm{I}}} / L_{B}$ galaxy DDO 154 by Kennicutt \& Skillman (2001) that shows the same trend for N/O. In both galaxies nitrogen from any past star formation events has had time to disperse, and there is no current burst of star formation to reduce the N/O ratio, neither galaxy having a particularly high current star formation rate (Skillman et al. 2003a; Kennicutt \& Skillman 2001, respectively), and like ESO 215-G?009 they may be considered quiescent galaxies. In fact, their current star formation rate and luminosity (which can be used as a rough estimate of the average past star formation rate; Tinsley 1980) are similar to what was found for ESO 215-G?009 in Paper I. This suggests that high H I mass-tolight ratio galaxies may have been forming stars at a low, constant rate over their lifetimes. In order to understand further the significance of this result, we would need to expand this study to spectra of $\mathrm{H}$ II regions in other high- $\mathcal{M}_{\mathrm{H}_{\mathrm{I}}} / L_{B}$ galaxies (especially ESO 215-G?009) and look at other ratios that may indicate the timing of star formation events. Metallicity may also be 
an important element in determining the fraction of baryons that remain in gas form (Taylor \& Webster 2005).

A close look at the plots of $\mathcal{M}_{\mathrm{H}_{\mathrm{I}}} / L_{B}$ versus $M_{B}$ (Figs. 1 and 11) shows that there is potentially an upper envelope to a galaxy's $\mathrm{H}$ I mass-to-light ratio at a given luminosity. Low-luminosity galaxies appear to be able to have a higher portion of their detectable baryons in the form of neutral hydrogen than galaxies around $L_{*}$, where the baryonic mass is dominated by stars even for the most gas-rich galaxies. What this suggests is that there is a minimum quantity of stars a galaxy will form that goes as a function of initial baryonic mass. Support for this idea can also be found in the theoretical work of Taylor \& Webster (2005). Whether or not a galaxy forms more than this minimum is likely to be a function of such factors as environment and the galaxy's dark matter properties.

Taylor \& Webster (2005) developed models to determine whether a neutral gas disk without stars (a "dark galaxy") could remain dynamically stable or whether some gas will collapse and form stars. They found that without an internal radiation field the majority of the gas in the disk will become gravothermally unstable, even for galaxies with very low baryonic masses (down to $5 \times 10^{6} \mathcal{M} \odot$ ). They also found that the fraction of unstable gas decreases as the baryonic mass decreases. This may provide an explanation as to why we see the slope in the upper envelope for $\mathcal{M}_{\mathrm{H}} / L_{B}$, the lower mass galaxies only having to convert a much smaller fraction of their baryons to stars in order to become stable. Galaxies such as ESO 215-G?009 and DDO 154 are close to our upper envelope, and they may define the extreme cases of galaxies that have formed only the minimum number of stars required in order to remain stable and that have not experienced any other events that may trigger star formation. In this way they might be used to distinguish between various models of galaxy collapse by defining the minimum star formation required for stability. We will further explore this possibility, and other properties that vary with $\mathcal{M}_{\mathrm{H}} / L_{B}$, with a larger sample of 37 latetype dwarf galaxies in an upcoming paper.

\section{CONCLUSIONS}

We obtained accurate optical CCD apparent magnitudes and $\mathrm{H}$ I flux densities for nine late-type dwarf galaxies and recalculated their $\mathrm{H}$ I mass-to-light ratios. The new $\mathcal{M}_{\mathrm{H}_{\mathrm{I}}} / L_{B}$ values are

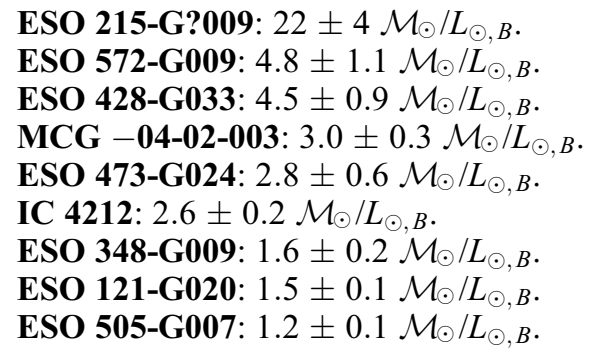

Many of these $\mathrm{H}$ I mass-to-light ratios are significantly below the initial estimates, due to inaccurate magnitude estimates in the literature. This strongly emphasizes the importance of having accurate observations in both the $\mathrm{H}_{\mathrm{I}}$ line and the optical. On the basis of the new H I mass-to-light ratio distribution, we conclude that genuine "dim" galaxies with high ratios $\left(\mathcal{M}_{\mathrm{H}_{\mathrm{I}}} / L_{B}>\right.$ $5 \mathcal{M}_{\odot} / L_{\odot, B}$ ) are rare in the local universe.

A previously uncataloged companion galaxy to ESO 121G020 was found at a projected distance of 3'. ATCA J061608574552 has an $\mathrm{H}$ I mass of $\sim 10^{7} \mathcal{M} \odot$ and an $\mathcal{M}_{\mathrm{H}} / L_{B}$ value of $2.2 \pm 0.3 \mathcal{M} \odot / L_{\odot, B}$. This was the only such companion detected and is well within the beam of the multibeam instrument used by the HIPASS survey. Despite our low-resolution $\mathrm{H}$ I observations, we were able to separate the galaxy ESO 505-G007 from its close neighbor ESO 505-G008 and determined H I flux densities of $21 \pm 3$ and $8 \pm 3 \mathrm{Jy} \mathrm{km} \mathrm{s}^{-1}$, respectively.

The best examples of high- $\mathcal{M}_{\mathrm{H}_{\mathrm{I}}} / L_{B}$ dwarf galaxies in the literature all have highly extended $\mathrm{H}$ I disks, are spatially isolated, and have normal baryonic content for their dynamical masses. The galaxies are not lacking the baryons to create stars but are underluminous, as they lack either the internal or external stimulation for further star formation. Future examination of element abundances within star formation sites of high- $\mathcal{M}_{\mathrm{H}}{ }_{\mathrm{I}} / L_{B}$ galaxies may reveal important clues about their star formation history. Recent observations (Skillman et al. 2003b; Kennicutt \& Skillman 2001) support the idea that high- $\mathcal{M}_{\mathrm{H}_{\mathrm{I}}} / L_{B}$ galaxies may have been forming stars at a low, constant rate over their lifetimes, as proposed in Paper I. There may be a minimum quantity of stars a galaxy will form that depends on the initial baryonic mass, which is supported by the theoretical work of Taylor \& Webster (2005). If this is true, then maybe high H I mass-to-light ratio galaxies have over their lifetimes only formed the minimum number of stars necessary to maintain the stability of their $\mathrm{H}$ I gas disk.

We are grateful for the assistance of Ken Freeman and Lister Staveley-Smith in this project, especially for their assistance with observations. We would like to thank Erwin de Blok for his help with various aspects of the $\mathrm{H}$ I data reduction and interpretation. We would also like to thank Marilena Salvo and Gayandhi de Silva for their observing assistance. Our thanks also go to the anonymous referee for useful comments, especially regarding the $\mathrm{H}$ i spectra. The $2.3 \mathrm{~m}$ telescope is run by the Australian National University as part of the Research School of Astronomy and Astrophysics. The Australia Telescope Compact Array and the Parkes Radio Telescope are part of the Australia Telescope, which is funded by the Commonwealth of Australia for operation as a National Facility managed by CSIRO. This research has made use of the NASA/IPAC Extragalactic Database, which is operated by the Jet Propulsion Laboratory, California Institute of Technology, under contract with the National Aeronautics and Space Administration. The Digitized Sky Survey was produced at the Space Telescope Science Institute under US government grant NAGW-2166, based on photographic data obtained using the UK Schmidt Telescope.
Begeman, K. G. 1989, A\&A, 223, 47

Begum, A., Chengalur, J. N., \& Karachentsev, I. D. 2005, A\&A, 433, L1

Bekki, K., Koribalski, B. S., \& Kilborn, V. A. 2005, MNRAS, 363, L21

Blanton, M. R., Lupton, R. H., Schlegel, D. J., Strauss, M. A., Brinkmann, J.,

Fukugita, M., \& Loveday, J. 2005, ApJ, 631, 208

Blanton, M. R., et al. 2003, ApJ, 592, 819

Carignan, C., \& Beaulieu, S. 1989, ApJ, 347, 760

Chung, A., van Gorkom, J. H., O’Neil, K., \& Bothun, G. D. 2002, AJ, 123, 2387

Côté, S., Freeman, K. C., Carignan, C., \& Quinn, P. J. 1997, AJ, 114, 1313

\section{REFERENCES}

Dalal, N., \& Kochanek, C. S. 2002, ApJ, 572, 25

de Vaucouleurs, G., de Vaucouleurs, A., Corwin, H. G., Buta, R. J., Paturel, G., \& Fouqué, P. 1991, Third Reference Catalogue of Bright Galaxies (New York: Springer)

Disney, M. J. 1976, Nature, 263, 573

Doyle, M. T., et al. 2005, MNRAS, 361, 34

Dwek, E. 1998, ApJ, 501, 643

Fouqué, P., Bottinelli, L., Durand, N., Gouguenheim, L., \& Paturel, G. 1990, A\&AS, 86, 473 
Hilker, M., Mieske, S., \& Infante, L. 2003, A\&A, 397, L9

Hirashita, H. 1999, ApJ, 522, 220

Hoffman, G. L., Lu, N. Y., Salpeter, E. E., Farhat, B., Lamphier, C., \& Roos, T. 1993, AJ, 106, 39

Izotov, Y. I., \& Thuan, T. X. 1999, ApJ, 511, 639

Jerjen, H. 2003, A\&A, 398, 63

Jerjen, H., Freeman, K. C., \& Binggeli, B. 1998, AJ, 116, 2873

Karachentsev, I. D., Karachentseva, V. E., Huchtmeier, W. K., \& Makarov, D. I. 2004, AJ, 127, 2031

Kauffmann, G., White, S. D. M., \& Guiderdoni, B. 1993, MNRAS, 264, 201

Kennicutt, R. C. 1989, ApJ, 344, 685

Kennicutt, R. C., \& Skillman, E. D. 2001, AJ, 121, 1461

Kilborn, V. A., et al. 2000, AJ, 120, 1342

Klypin, A., Kravtsov, A. V., Valenzuela, O., \& Prada, F. 1999, ApJ, 522, 82

Koribalski, B. S., et al. 2004, AJ, 128, 16

Kraan-Korteweg, R. C., \& Huchtmeier, W. K. 1992, A\&A, 266, 150

Landolt, A. U. 1992, AJ, 104, 340

Lauberts, A., \& Valentijn, E. A. 1989, The Surface Photometry Catalogue of the ESO-Uppsala Galaxies (Garching: ESO)

Maddox, S. J., Efstathiou, G., Sutherland, W. J., \& Loveday, J. 1990, MNRAS, 243, 692

Martin, C. L., \& Kennicutt, R. C., Jr. 2001, ApJ, 555, 301

Minchin, R., et al. 2005, ApJ, 622, L21

Monet, D. G., et al. 2003, AJ, 125, 984

Moore, B., Ghigha, S., Governato, F., Lake, G., Quinn, T., Stadel, J., \& Tozzi, P. 1999, ApJ, 524, L19
Oosterloo, T., \& van Gorkom, J. 2005, A\&A, 437, L19

Paturel, G., et al. 1997, A\&AS, 124, 109

Richter, O.-G., \& Huchtmeier, W. K. 1987, A\&AS, 68, 427

Roberts, M. S., \& Haynes, M. P. 1994, ARA\&A, 32, 115

Ryan-Weber, E., et al. 2002, AJ, 124, 1954

Ryder, S. D., \& Koribalski, B. S. 2004, in IAU Symp. 217, Recycling Intergalactic and Interstellar Matter, ed. P.-A. Duc, J. Braine, \& E. Brinks (San Francisco: ASP), 44

Ryder, S. D., et al. 2001, ApJ, 555, 232

Schlegel, D. J., Finkbeiner, D. P., \& Davis, M. 1998, ApJ, 500, 525

Shapiro, P. R., Iliev, I. T., \& Raga, A. C. 2004, MNRAS, 348, 753

Skillman, E. D., Côté, S., \& Miller, B. W. 2003a, AJ, 125, 593 2003b, AJ, 125, 610

Taylor, E. N., \& Webster, R. L. 2005, ApJ, 634, 1067

Tinsley, B. M. 1980, Fundam. Cosmic Phys., 5, 287

Toomre, A. 1964, ApJ, 139, 1217

Trentham, N., \& Tully, R. B. 2002, MNRAS, 335, 712

van Zee, L., Haynes, M. P., Salzer, J. J., \& Broeils, A. H. 1997, AJ, 113, 1618

Verde, L., Oh, S. P., \& Jimenez, R. 2002, MNRAS, 336, 541

Warren, B. E., Jerjen, H., \& Koribalski, B. S. 2004, AJ, 128, 1152 (Paper I)

Widrow, L. M., \& Dubinski, J. 1998, ApJ, 504, 12

Young, L. M., van Zee, L., Lo, K. Y., Dohm-Palmer, R. C., \& Beierle, M. E. 2003, ApJ, 592, 111

Zwaan, M. A., et al. 2003, AJ, 125, 2842 